Check for updates

Cite this: RSC Adv., 2017, 7, 33642

Received 9th May 2017

Accepted 12th June 2017

DOI: 10.1039/c7ra05256a

rsc.li/rsc-advances

\section{A retrospect on the role of piezoelectric nanogenerators in the development of the green world}

\author{
Ani Melfa Roji M, (D)*a Jiji $G^{b}$ and Ajith Bosco Raj T (D)*b \\ This paper gives a detailed report of the evolution and potential applications of piezoelectric \\ nanogenerators (PENGS). Various configurations, together with the operating principles and techniques \\ used for the fabrication of PENGs, are discussed. A brief overview of the wide range of materials used for \\ the design of PENGs that exhibit piezoelectric properties is also reported here. The optimization \\ parameters to be considered while designing a PENG are also presented. PENGs are used in several \\ application areas, which are collectively summarized.
}

\section{Introduction}

Self-powered devices are becoming ubiquitous and are receiving increasing interest, because they dispense with the need for the replacement of chemical batteries. As the world has been migrating towards portability and miniaturization, the nanogenerator (NG) has become a demanding area of research. Size, complexity and power consumption are greatly reduced because of the development of such energy-scavenging devices. Hence, there is a great need to develop sustainable self-sufficient eco-

${ }^{a}$ Department of Electronics and Communication Engineering, PSN College of Engineering and Technology, Tirunelveli, Tamil Nadu, India - 627152. E-mail: animelfarojim@gmail.com

${ }^{b}$ Department of Electronics and Communication Engineering, PSN College of Engineering and Technology, Tirunelveli, Tamil Nadu, India - 627152. E-mail: ajithboscoraj@gmail.com friendly nanoscale power sources as alternative forms of green energy owing to the life-threatening effects of battery disposal and the fossil energy crisis. ${ }^{1}$ The abundant renewable energy resources, such as solar, wind, thermal, pressure, human power, vibrations, etc., are generally underutilized for energy harvesting. NGs can be developed using three different approaches, namely, triboelectric, ${ }^{2}$ piezoelectric ${ }^{3}$ and pyroelectric. ${ }^{4}$ Among these, the piezoelectric approach is widely used, because vibrations attracted many researchers as a renewable power source, owing to its excellent environmental adaptability and high robustness. ${ }^{5}$ A large number of vibrations, such as human motion, breathing, heartbeats, ${ }^{6}$ walking, low-frequency seismic vibrations, running automobiles, tides, waves, the wind, blood flow, mechanical triggering, rotating tires, acoustic waves, flowing water, muscle stretching, ${ }^{7,8}$ tiny clicks of the fingers, ${ }^{5}$ etc., are available from the surrounding environment

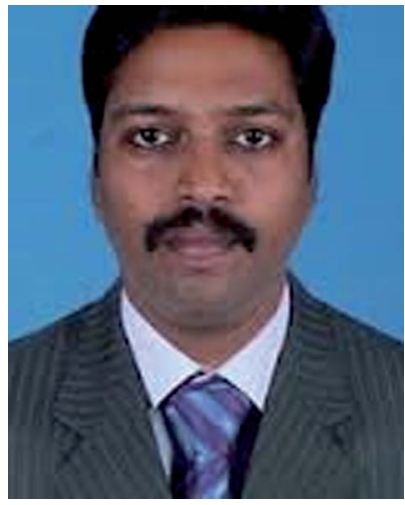

Dr Ajith Bosco Raj $T$ received a B.E. degree in Electronics and Communication Engineering and M.E. degrees from St. Xavier's Catholic College of Engineering, Kanyakumari, India. He completed his Ph.D. in the College of Engineering, Guindy, Chennai. $\mathrm{He}$ is currently working as a professor in PSN College of Engineering and Technology, Tirunelveli, India. He has published several research articles in reputed high-impact journals. His research interests include electrical engineering, power converters, wireless sensor networks, embedded systems design and robotics. 
but are wasted in our daily life. A PENG is a nanoscale energy harvester that converts kinetic energy from mechanical vibrations in the ambient environment into a usable form of electrical energy using a piezoelectric material. The first PENG was fabricated using zinc oxide nanowire (NW) arrays, which produce a strain field, and charge separation takes place owing to the piezoelectric and semiconducting properties of $\mathrm{ZnO}$ when it is bent by an atomic force microscopy (AFM) tip; its efficiency is estimated to be $17 \%$ to $30 \% .^{9}$

Nanoscale energy harvesting is an emerging field in which efficient energy conversion is a challenging task. Various research studies have been carried out to increase the energy conversion ratio of such harvesters. Nanoscale energy harvesters greatly reduce the size of power sources and are hence very helpful in the development of many nanoelectromechanical systems (NEMS) for various applications such as optoelectronics, ${ }^{10}$ resonators, ${ }^{11}$ biosensors, ${ }^{12}$ and so on. They also pave the way for many emerging fields such as smart consumer electronics (mobiles, laptops), microelectromechanical systems (MEMS), defense technology (spy robots, high-speed wireless sensors), biomedicine (implantable devices), etc. This emerging technology has a number of other advantages such as low expenditure in manufacturing and synthesis, high robustness, reliability, eco-friendliness, and so on. The size and complexity of devices have been reduced by replacing the need for conventional bulk batteries that have to be replaced or recharged periodically by these PENGs. They are a promising solution for the development of very compact self-powered portable devices, which makes human life more convenient.

NGs have drawn the interest of many researchers because of their outstanding performance in MEMS/NEMS applications. However, the conversion efficiency and generated power of early-stage NGs are too low to be utilized. Therefore, several research works have been performed with great efforts to improve the performance of NGs by utilizing diverse piezoelectric materials with better piezoelectric properties, ${ }^{\mathbf{1 3 - 1 5}}$ novel NG designs ${ }^{16,17}$ and hybrid NG designs. ${ }^{18,19}$

This paper aims to provide a clear idea of PENGs, including their evolution and involvement in the field of nanotechnology. This paper is organized as follows. The basic operating principle of NGs is described in Section II. In Section III various structural configurations of NGs are reported. Various fabrication techniques used for the synthesis of nanostructures are discussed together with their limitations in Section IV. Section $\mathrm{V}$ provides a detailed description of different materials used for the design of PENGs. The factors that improve the efficiency of piezoelectric energy-scavenging devices are explained in Section VI. The different types of available hybrid energy harvester are reported in Section VII. The use of piezoelectric nanoscale harvesting in a wide range of areas is summarized in Section VIII. Section IX concludes the in-depth review of the paper.

\section{Modes of operation}

PENGs have two basic modes of operation depending upon the direction of the application of force.

\section{A. Force exerted parallel to NG axis}

When a force is applied to the tip surface of a vertically grown nanostructure (NS), uniaxial compression of the structure takes place and an electric dipole moment is created. The operation of a PENG with a force applied parallel to the axis of the nanogenerator is illustrated in Fig. 1.

A negative electric potential is created at the tip of the NS owing to a direct piezoelectric effect, which raises the Fermi level at the tip. A positive electric potential is created at the other tip owing to the flow of electrons from the top tip to the bottom. The periodic peak in potential is responsible for the formation of a Schottky contact, which is due to the piezotronic effect, i.e., the coupling of semiconducting and piezoelectric properties. $^{20}$ The tip potential is maintained with the help of the Schottky contact, which blocks the flow of electrons through the NS and acts as a charge pump to pass electrons from the top to the bottom through the external circuit. Electrons accumulate at the bottom until an equilibrium state is attained. When the external force is removed, the induced piezoelectric effect instantly vanishes. The positive potential at the bottom tip of the NS is neutralized because of the migration of accumulated

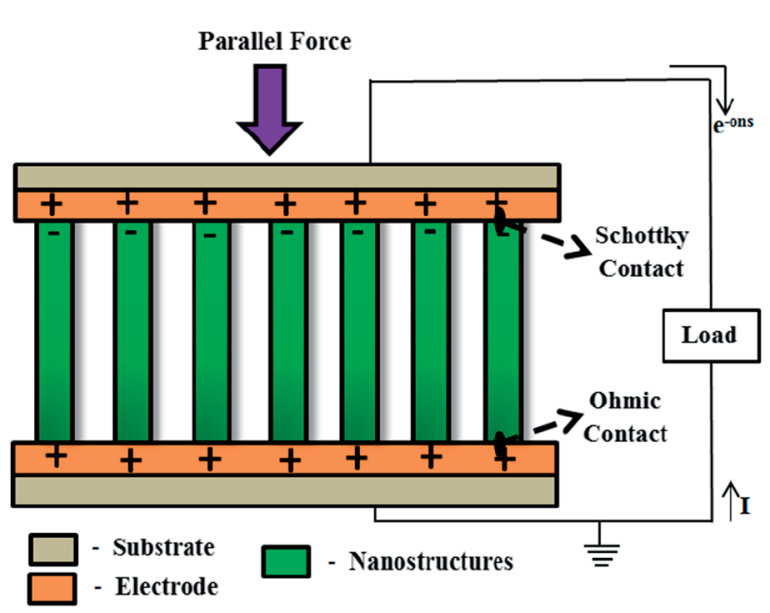

(a)

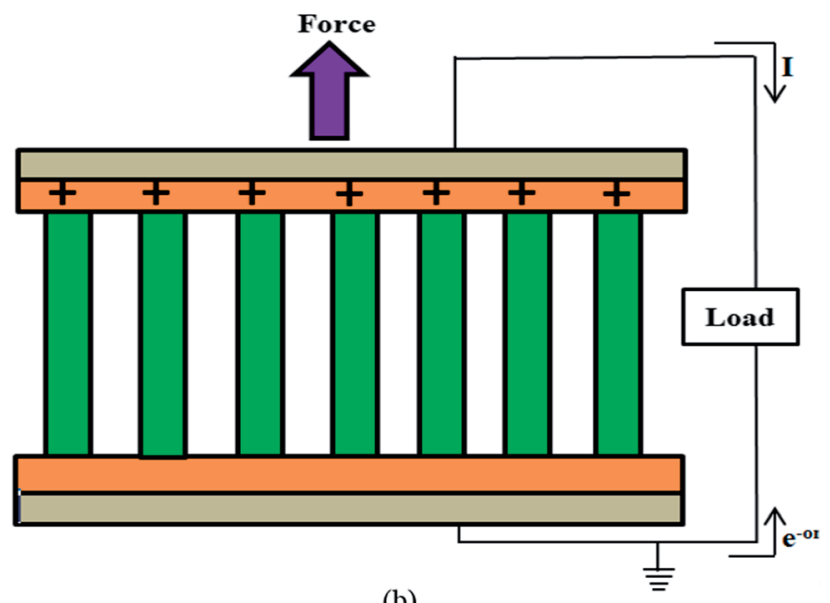

(b)

Fig. 1 Schematic of PENG. (a) After the application of a force parallel to the NG axis. (b) After the removal of the force. 
electrons from the bottom to the top electrode of the NS via the external circuit. A voltage peak in the opposite direction is obtained and hence an alternating current (AC) is generated.

\section{B. Force exerted perpendicularly to NG axis}

When a force is applied to the laterally moving tip of a PENG, the NS undergoes deformation, which leads to the generation of an electric field. Fig. 2 shows a schematic of a PENG when a force is exerted perpendicularly to the direction of the axis of the nanogenerator. One portion at the tip of the NS undergoes expansion, while the other portion undergoes compression. The compressed end exhibits a positive strain and a positive electric potential, whereas the other end exhibits a negative strain as well as a negative electric potential. Hence, electrical energy is distributed at the ends of the NS. The ohmic contact that is formed between the bottom metal electrode and the NS causes neutralization of the electric field generated at the tip of the NS.

The Schottky contact that is formed between the top metal electrode and the tip of the NS (Fig. 3) is responsible for the generation of an electric current because of its rectifying characteristics. ${ }^{9}$ The nanogenerator becomes conductive only when the top metal electrode is in contact with the region of negative potential at the tip of the NS, owing to the flow of electrons from the tip surface to the top electrode. This leads to the generation of a direct current (DC). In 2005, Hasegawa et al. used an aluminium $(\phi=4.08 \mathrm{eV})$ disc in their n-type ZnO-based nanorod (NR) $\left(E_{\mathrm{a}}=4.5 \mathrm{eV}\right)$ design in order to create an ohmic contact and also to increase the conductivity of the electrode. ${ }^{21}$ Mead et al. (1965) showed that an ohmic contact has the ability to eliminate the barrier at an $\mathrm{Al}-\mathrm{ZnO}$ interface and thus enables free movement of electrons on both sides, i.e., from the electrode to the $\mathrm{ZnO}$ NRs and vice versa. $^{22} \mathrm{Cu}(\phi=4.53-5.10 \mathrm{eV})$ was chosen as the bottom electrode by Chengkun et al. (2010) to create a Schottky contact at a $\mathrm{Cu}-\mathrm{ZnO}$ interface, which dominates the entire conduction process. ${ }^{23}$

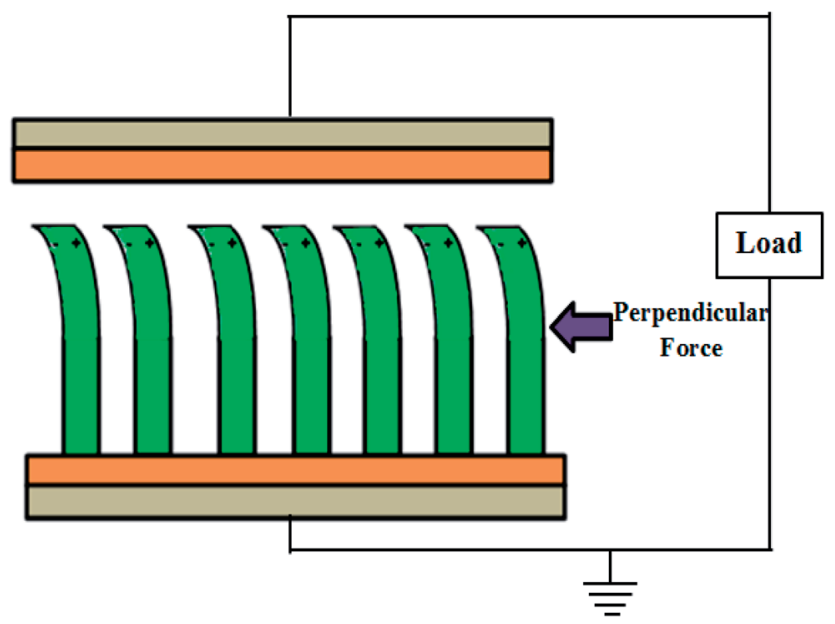

Fig. 2 Schematic of PENG after the application of a force perpendicularly to the NG axis.
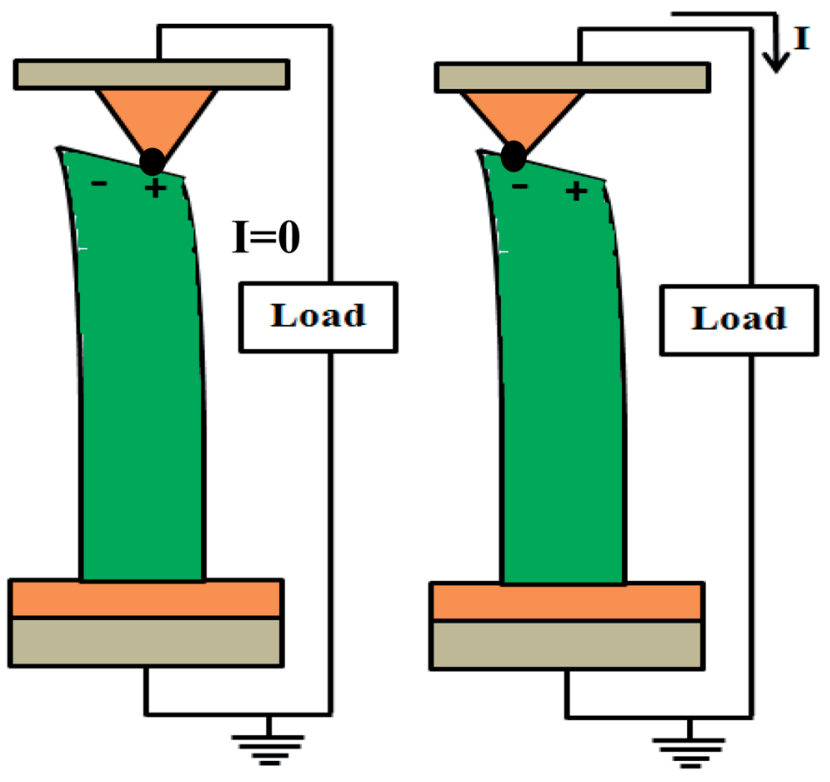

Fig. 3 Operating principle of PENG and formation of a Schottky contact with the application of a perpendicular force.

\section{Structural configuration}

The performance of PENGs can be improved from the point of view of materials in numerous ways. Extensive studies have been performed in basic science and materials science by chemists and materials scientists and have been utilized in various practical applications. NGs are broadly categorized into four types on the basis of their configuration, namely, single-wire generators (SWGs), lateral nanowire integrated nanogenerators (LINGs), nanocomposite electrical generators (NEGs) and vertical nanowire integrated nanogenerators (VINGs).

\section{A. Single-wire generator (SWG)}

The SWG is a configuration that was widely used in the early stages of development of NGs. It consists of two parts, namely, a laterally aligned NS and a flexible substrate. The NS is incorporated into the substrate in order to achieve the electrical energy-generating potential of the piezoelectric material. When the SWG undergoes stretching, a piezoelectric potential is created at the tip of the NW. The Schottky contact prevents the flow of electrons through the external circuit. The charging and discharging mechanisms of the NG are maintained with the help of the NW by controlling the backwards and forwards movement of electrons via the external circuit. Hence, the NW behaves like a capacitor or charge pump that drives the movement of electrons depending upon the deformation of the nanostructure. ${ }^{24}$ The individual piezoelectric NS is subjected to pure tensile strain because the diameter of the NS is very much less than the thickness of the substrate film in most cases.

\section{B. Lateral nanowire integrated nanogenerator (LING)}

The LING is an elaboration of the SWG. It is a two-dimensional configuration. It comprises three sections, namely, a base 


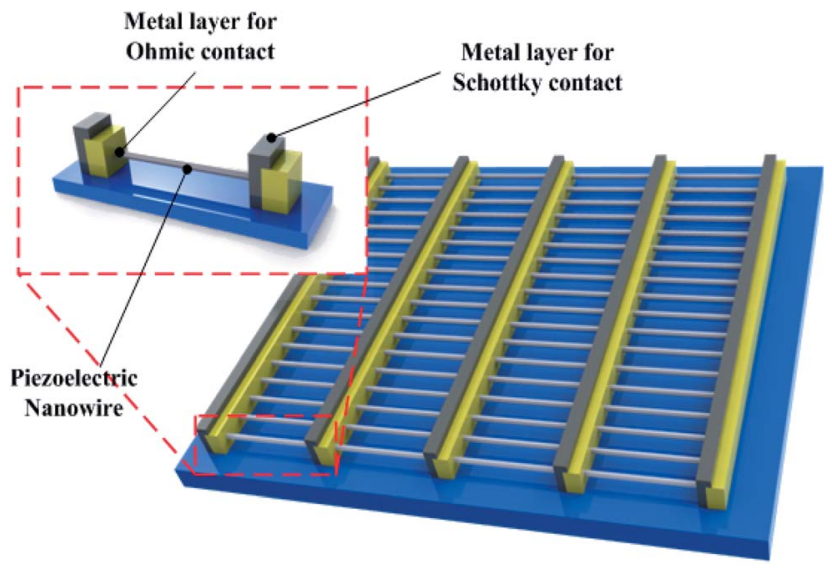

Fig. 4 Basic structure of LING.

electrode, laterally grown piezoelectric NSs and a top metal electrode, as shown in Fig. 4. The first metal electrode is used for establishing the Schottky contact, whereas the second metal electrode is responsible for the creation of the ohmic contact.

The AC signal is generated using this configuration. The voltage generated by an LING can be increased by arranging a large number of LINGs in series. This series arrangement of LINGs provides improved performance, which makes it suitable for scavenging large-scale power from tides, waves and the wind.

\section{Nanocomposite electrical generator (NEG)}

The NEG is a three-dimensional configuration. It consists of three parts, namely, metal plate electrodes, vertically grown piezoelectric nanostructures and a polymer matrix, which fills the spaces between the piezoelectric NSs, as shown in Fig. 5 .

A generator with the NEG configuration, which was composed of an array of zinc oxide nanowires embedded in a finite epoxy matrix, was initially proposed by Momeni et al. in $2010 .^{25}$ Modeling of an NEG was provided by Momeni et al. in 2012 using continuum mechanics and Maxwell's equations. ${ }^{26}$ The effect of the aspect ratio of the ZnO NWs on the electric potential under axial loading was also considered, and the adhesion between the ZnO NWs and the polymer matrix was observed to be perfect, with effective linear piezoelectric behavior.

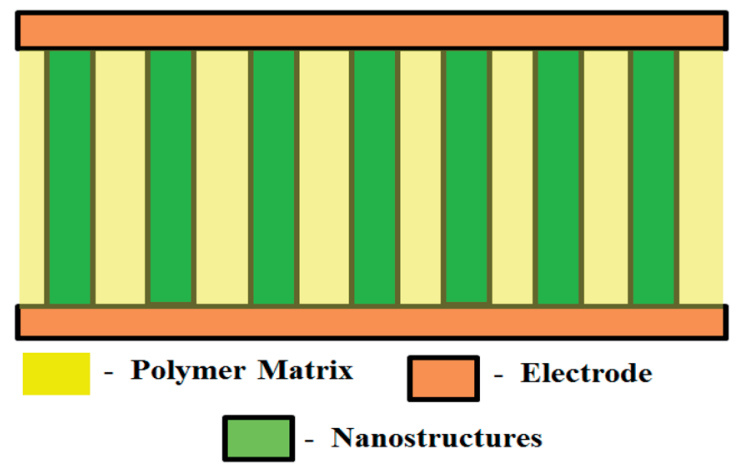

Fig. 5 Schematic diagram of NEG.

\section{Vertical nanowire integrated nanogenerator (VING)}

The VING is a three-dimensional configuration. It consists of three layers, namely, a base electrode, vertically grown piezoelectric NSs and a counter electrode. A general schematic diagram of a VING is shown in Fig. 1. Different synthesis methodologies are followed for the growth of piezoelectric NSs on the base electrode. The basic configuration of the VING was introduced by Zhong et al. in 2006. Wang et al. developed an initial VING in which the deformation of a single vertical ZnO nanowire was induced by AFM in 2007. ${ }^{27}$ The counter electrode together with the periodic surface grating electrode are in partial contact with the NS tips and are utilized in resemblance to AFM tip arrays as a moving electrode. External vibrations result in the in-plane and out-of-plane motion of individual NSs, which causes deformation of the structure, and hence a distribution of potential is created across each NS. The counter electrode is integrated with the tips of the NSs in full or partial mechanical contact mode.

The topology of the counter electrode plays a major role in the case of a VING with a partial contact, in which a DC signal is generated with no external rectifier because of its on/off switching characteristics. The deformation induced by the flat counter electrode moving in in-plane mode is not sufficient. Choi et al. (2009) developed a transparent electrode with a bowl shape with anodized aluminium by means of electroplating technology. ${ }^{28}$ The transparent counter electrode with a networked single-walled carbon nanotube (SWNT) was developed on a flexible substrate and exhibited exceptional energy conversion efficiency. ${ }^{29}$

A VING with full mechanical contact between the tips of the NSs and the counter electrode seems to perform efficiently when a force is exerted in the vertical direction of the NSs. ${ }^{20} \mathrm{~A}$ VING with full mechanical contact generates an AC signal similar to that of an LING.

The VING configuration is generally adopted for harvesting mechanical energy owing to its low cost and easy synthesis, ${ }^{30}$ whereas an LING requires expensive steps such as the sputtering of an Au or Pt electrode layer on substrates, and lithographic lift-off processes are also involved in providing a mask to achieve the patterned growth of ZnO NSs. Similarly, NEGs require a complicated filling process, which uses expensive filling solutions to fill the spaces between NSs. ${ }^{31,32}$

In 2008, Qin et al. proposed a fabric-like configuration in which NSs are formed vertically in a radial direction on two microfibers, which are twisted together to form an NG. ${ }^{8}$ One of the microfibers is kept static, while the other is allowed to move. A voltage is generated by the deformation of NSs on the stationary microfiber owing to stretching of the movable microfiber. This performs in a similar way to VINGs with partial mechanical contact and leads to the generation of a DC electrical signal.

\section{Synthesis methodologies}

Research on PENGs has been carried out for over a decade. The size and complexity of piezoelectric generators are greatly 
reduced by miniaturization with limited efficiency. The major limitations in the synthesis of different nanostructures are their low quantity and reduced physical stability. Several synthesis techniques have been adopted for the synthesis of various NSs to overcome these limitations. ${ }^{28,29}$

The techniques used for the synthesis of nanomaterials are broadly categorized into two types, namely, top-down approaches and bottom-up approaches.

\section{A. Top-down approaches}

Top-down approaches involve the fabrication of NSs from a bulk piece of material and are suitable for large-scale production. They include nanolithography, ${ }^{33}$ anodization, ${ }^{34}$ laser machining ${ }^{35}$ and dry etching. ${ }^{36}$

The most commonly used top-down approach is nanolithography. Depending on the desired minimum size of the NS, lithography can be employed using light (optical or photolithography), ${ }^{37}$ electrons (e-beam lithography), ${ }^{38}$ ions (i-beam lithography) ${ }^{39}$ or X-rays (X-ray lithography), ${ }^{40}$ as shown in Fig. 6. This technique has lost its importance because the fabrication of NSs smaller than $100 \mathrm{~nm}$ is difficult owing to diffraction effects, costly photolithographic tools are involved and the mask has to be perfectly aligned with the wafer pattern for efficient synthesis. Dip-pen nanolithography (DPN) operates in positive printing mode and deposits molecules on a surface with the help of a small tip. ${ }^{41}$ It can fabricate NSs with sizes of less than $40 \mathrm{~nm}$.

Anodization is used to grow a thin, dense oxide barrier of uniform thickness on various metals under specific process conditions. ${ }^{43}$

Dry etching is carried out in the gas phase to remove materials without involving any aqueous solutions. This technique can be either purely chemical (plasma etching, PE) ${ }^{44}$ purely physical (ion beam milling, IBM) ${ }^{45}$ or a combination of both (reactive ion etching, RIE). ${ }^{46}$

These time-consuming costly processes involve many technological limitations, and hence top-down approaches are no longer widely used.

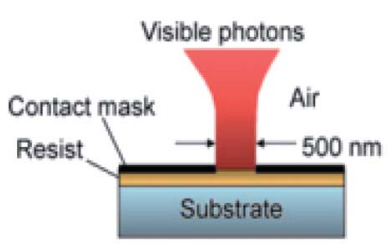

PHOTOLITHOGRAPHY

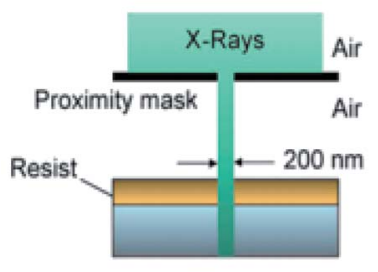

X-Ray LITHOGRAPHY

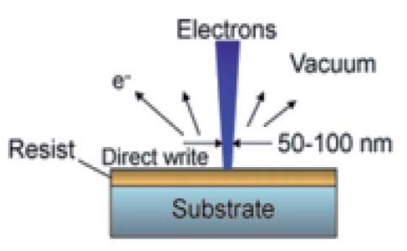

E- beam LITHOGRAPHY

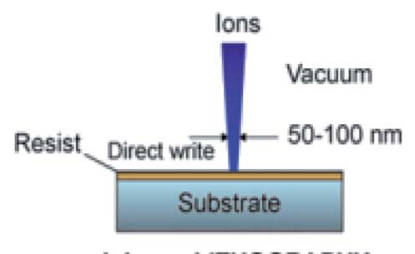

|- beam LITHOGRAPHY
Fig. 6 Comparison of the available lithography techniques. ${ }^{42}$

\section{B. Bottom-up approaches}

Bottom-up approaches involve the synthesis of NSs by the selfassembly of atoms or molecules with the help of chemical reactions. The most commonly utilized bottom-up approaches are sol-gel processing, ${ }^{47}$ laser pyrolysis, ${ }^{48}$ chemical vapor deposition (CVD) ${ }^{49,50}$ plasma or flame spraying synthesis, ${ }^{51}$ hydrothermal methods, ${ }^{52-54}$ chemical bath deposition (CBD) ${ }^{55,56}$ and aqueous chemical growth (ACG). ${ }^{30,57}$

CVD processes are able to produce stable NSs with high crystal quality and can be easily controlled for relatively largescale production. Owing to the limited size of the growth platforms and silicon wafers, the NSs grown in long horizontal quartz tubes were insufficient for designing an NG. Hence, an alternative approach was presented by Mohiuddin et al. (2016), whereby large-scale production can be easily achieved by using the inner side of a horizontal quartz tube as the growth platform, and thus an NG has been developed by a tube-in-tube chemical vapor deposition (CVD) technique with a maximum output voltage of $0.7 \mathrm{~V} .{ }^{31} \mathrm{~A}$ schematic of a microwave-assisted CVD process is shown in Fig. 7.

Hydrothermal methods have attracted many scientists because of their low cost, low-temperature environment below $100{ }^{\circ} \mathrm{C},{ }^{3}$ large-scale production and substrate flexibility. ${ }^{32} \mathrm{Xu}$ et al. (2013) synthesized ZnO NWs by a hydrothermal method and observed a high concentration of native defects, ${ }^{59}$ which lead to unfortunate optical properties such as the suppression of UV emissions. ${ }^{32}$ The density and morphology of the grown NWs were controlled in this technique without using a catalyst. Many NSs such as ZnO NAs ${ }^{60}$ and KNKH NRs ${ }^{61}$ were successfully synthesized using this cheap method. Mohiuddin et al. (2016) observed that the hydrothermal aqueous solution may lead to the production of unstable NSs with larger structures owing to the recombination, aggregation and accumulation of NSs. ${ }^{31}$ The time-consuming hydrothermal approach still maintains its position owing to the high crystallinity of its nanomaterial product.

CBD has become an attractive synthesis method because it provides better performance while using flexible substrates and cost-efficient fabrication and requires moderate temperatures. ${ }^{62-66} \mathrm{~A} \mathrm{ZnO}$ nanorod array was grown on a ZnO-seeded FTO glass substrate using CBD by Chou et al. (2016). ${ }^{67}$ The experimental setup used for CBD techniques is shown in Fig. 8.

The ACG technique is simple and less expensive, operates at low temperatures and is less hazardous. Zafar et al. (2017) used

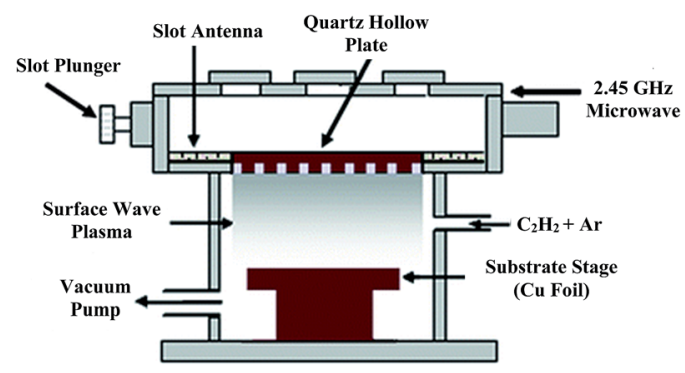

Fig. 7 Schematic of deposition of graphene film at low temperatures using a microwave-assisted surface-wave plasma chemical vapor deposition technique. ${ }^{58}$ 


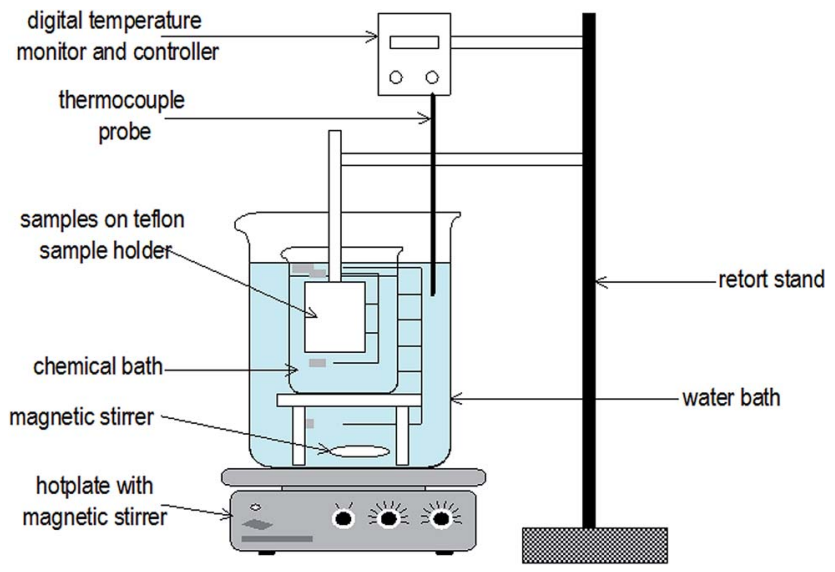

Fig. 8 Experimental setup used for chemical bath deposition. ${ }^{68}$

the aqueous chemical growth (ACG) technique to grow $\mathrm{ZnO}$ NSs on an Al-coated substrate. ${ }^{69}$ Zinc nitrate hexahydrate $\left[\mathrm{Zn}\left(\mathrm{NO}_{3}\right)_{2} \cdot 6 \mathrm{H}_{2} \mathrm{O}\right]$ solutions of equimolar concentrations were used for the growth of the NSs. This cost-efficient technique is able to synthesize controlled and well-aligned ZnO NSs at low temperatures. Ahmad et al. (2017) presented the controlled morphological growth of ZnO NWs in a catalyst-free environment by adjusting the physical growth parameters. ${ }^{70}$

Sol-gel processing is mainly used for the production of inorganic oxide materials using low-temperature wet chemistry techniques. This process is mainly employed in the production of thin films, because the deposition of nanomaterials on a substrate occurs at relatively low temperatures. ${ }^{71}$ The step-bystep procedure involved in the sol-gel synthesis of nanoparticles is depicted in Fig. 9. The sol-gel method has gained wide interest because of its simple procedure, low processing temperature, molecular homogeneity, large-area coatings, and inexpensive equipment, as well as low processing costs and better photocatalytic properties. ${ }^{72}$ Madou et al. (2002) fabricated lead zirconium titanate (PZT) ${ }^{73}$ and created various forms of ceramic or glass materials at low temperatures using sol-gel techniques. This is a versatile and less expensive approach. The limitations of sol-gel and plasma spraying techniques were stated by Mahtab et al. in 1995 to be a lack of adhesion of the coating to the substrate, non-uniformity of the thickness of the deposited layer and difficulties in maintaining structural integrity and the coating composition. ${ }^{74}$

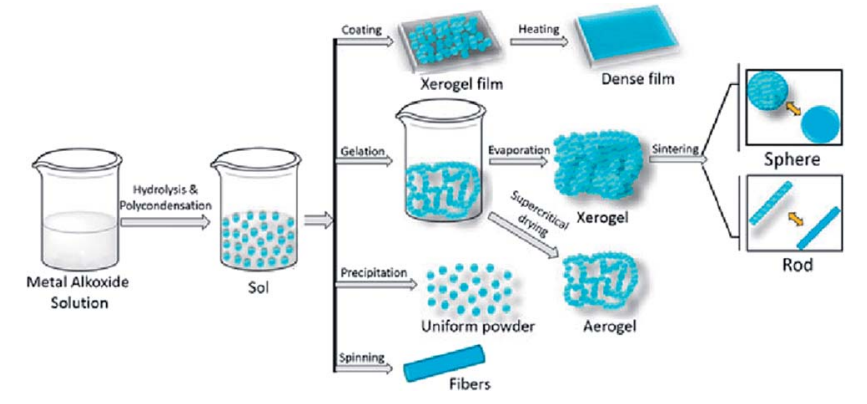

Fig. 9 Schematic illustration of sol-gel processes. ${ }^{75}$
Khan et al. (2016) successfully synthesized starch-protected zero-valent copper $(\mathrm{Cu})$ nanoparticles of an average crystallite size of $28.73 \mathrm{~nm}$ by a solution-phase approach called chemical reduction. ${ }^{76}$ Hasegawa et al. (2005) suggested that a photoreduction method was employed to synthesize Au nanoparticles, which were adsorbed onto a $\mathrm{ZnO}$ surface spontaneously owing to electrostatic attraction. ${ }^{21}$

Laser pyrolysis uses an IR laser for the decomposition of materials by heating gas-phase reactants in an oxygen-poor environment. Herlin et al. suggested that this technique is able to produce extremely pure spherical nanoparticles of small diameters with a low level of agglomeration. ${ }^{77}$ Laser ablation is a similar technique used for the production of $\mathrm{CNTs}^{78}$ and NWs. ${ }^{79}$ Schematics of the pyrolysis and electrospraying methods are provided in Fig. 10.

A thin-film piezoelectric nanogenerator can be fabricated by coating a nanomaterial solution on the surface of a suitable substrate. This process is called seed layer deposition. Seeding is the essential phase of nanogenerator design and can be performed by many techniques such as atomic layer deposition (ALD), ${ }^{53}$ RF sputtering, ${ }^{52,55,81}$ magnetron sputtering ${ }^{82}$ and spin coating, ${ }^{54}$ as shown in Fig. 11.

Electrospinning ${ }^{85-87}$ is a convenient method for preparing nanogenerators without further treatment. It has been proven that the physicochemical properties and electrical output of electrospun fibers strongly depend on the processing conditions, ${ }^{88,89}$ as illustrated in Fig. 12 and 13.

A liquid coating solution is prepared by mixing the assynthesized nanomaterials with a suitable solvent. In a dip coating process, a substrate is dipped into the prepared coating solution. After a particular time, the coated substrate is pulled out at a controlled speed. The thickness of the coating generally depends on the fluid viscosity, fluid density, and surface tension in such a way that the thickness of the coating increases linearly with the withdrawal speed. High-quality films with uniform coatings are produced by means of this procedure, but it requires a clean environment and precise control. A schematic of dip coating is shown in Fig. 14. Yunfeng et al. (1997) reported a sol-gel-based dip coating method for the fast and uninterrupted synthesis of mesoporous thin films on a solid substrate. ${ }^{92}$ Minbaek et al. (2012) designed an NG based on zinc oxide with a uniform surface and radially aligned dense $\mathrm{ZnO}$ NWs using a dip coating procedure. ${ }^{72}$ The entire zinc oxide NWs were completely immersed in a piezoelectric polymer (namely, PVDF) and drawn out slowly at a velocity of $\sim 5 \mathrm{~mm} \mathrm{~s}^{-1}$. Largescale batch processing is carried out using a dip coating process and finds applications in large laboratories, as well as in lens manufacturing industries.

Spin coating is the principal procedure used for seed layer deposition. In this process, a horizontally mounted substrate on a rotating platform rotates at high speed. The coating solution is sprayed over the spinning substrate, as depicted in Fig. 15. The thickness of the coating layers is closely dependent upon the spinning speed, whereby high-speed rotation leads to a thin, uniform coating and vice versa. This is a less time-consuming process and results in the fabrication of thin films with very high uniformity, even over a curved surface. Coating is carried 
(a)

(b)

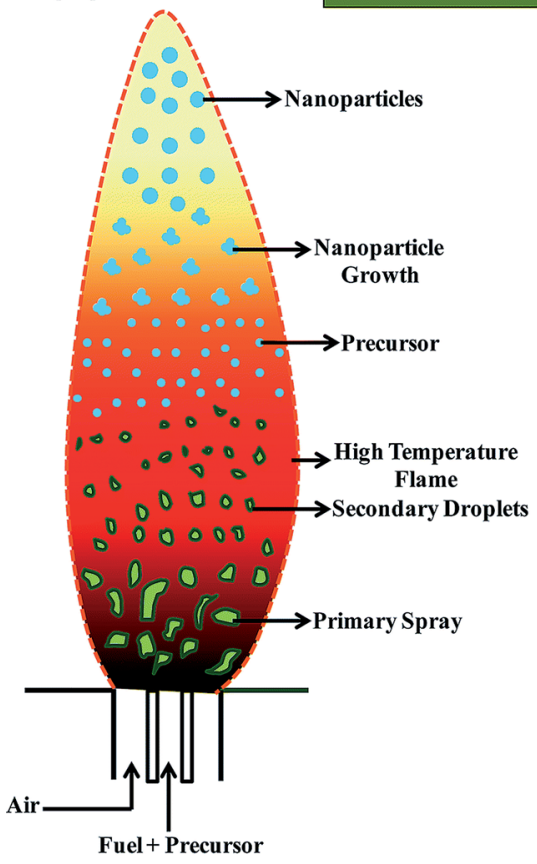

Confinement Gas
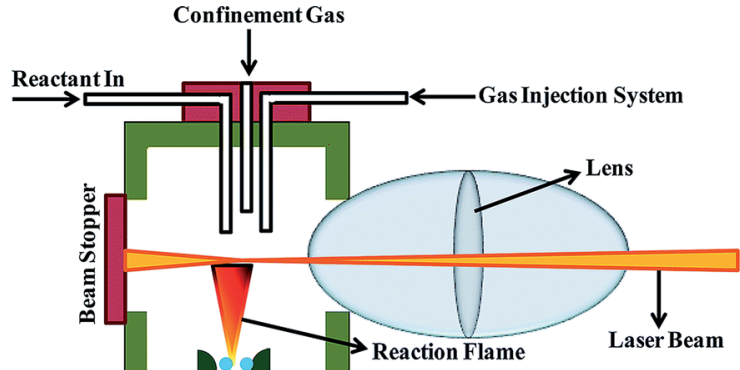

$\rightarrow$ Nanoparticles

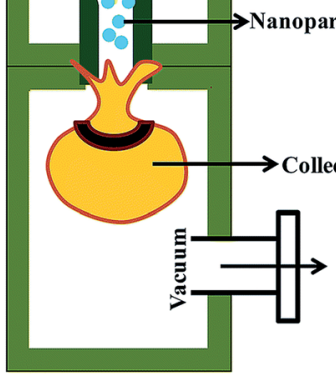

(c)

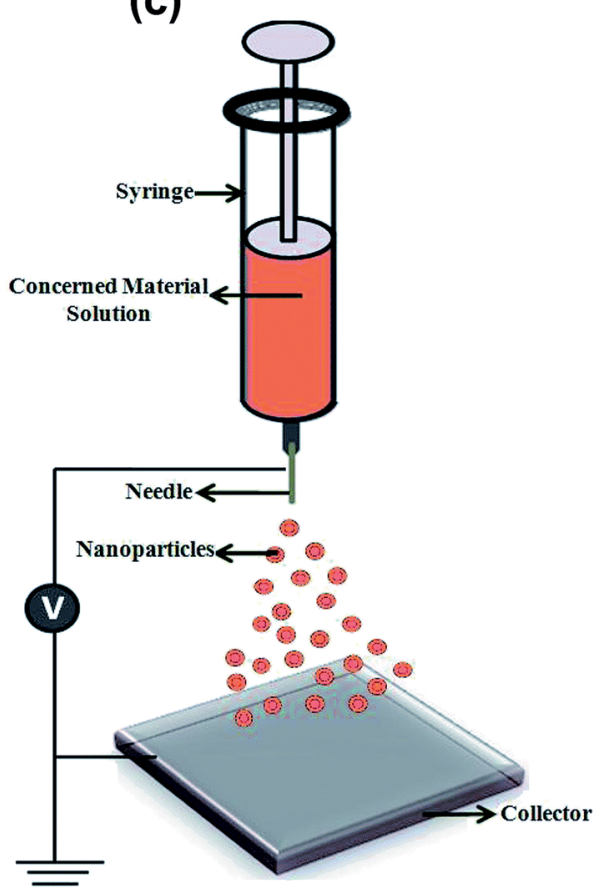

Fig. 10 Schematic diagrams of (a) laser pyrolysis, (b) flame spray pyrolysis and (c) an electrospraying technique for the synthesis of nanoparticles. ${ }^{80}$

out on a single area by means of spin coating, whereas multiple areas can be coated simultaneously by dip coating. Chou et al. (2016) deposited a ZnO seed layer on indium tin oxide (ITO) or fluorine-doped tin oxide (FTO) glass using this method and achieved dense deposition of the seed layer on glass. ${ }^{67}$

Seed layer annealing was found to be a crucial phase in the growth of nanowires. An array of $\mathrm{ZnO}$ nanorods was synthesized on ZnO seeds by Chou et al. in 2016, who observed that the recrystallization of $\mathrm{ZnO}$ grains and the growth of nanorods were improved whereas the ohmic series resistance $\left(R_{\mathrm{s}}\right)$ and the grain boundaries between $\mathrm{ZnO}$ grains were reduced at high annealing temperatures. ${ }^{67}$ After the annealing treatment, some of the atoms on the surface became more active, which resulted in the formation of a rough surface. ${ }^{59}$
Piezoelectric materials normally consist of randomly oriented microscopic electric dipoles, which result in zero net polarization. Owing to their minimal polarization, a negligible or weak piezoelectric effect is experienced when such materials are stressed by an external mechanical force. The material has to undergo a poling process (Fig. 16) in order to acquire or enhance its piezoelectric sensitivity. Poling is a process whereby the dipoles are forced to orient themselves in a prescribed direction by the application of an external electric field. Most of the dipoles follow the orientation even after the removal of the electric field.

When a mechanical force is applied across the poled piezoelectric material, the material is polarized and a voltage is generated owing to the accumulation of charge on the surface of 


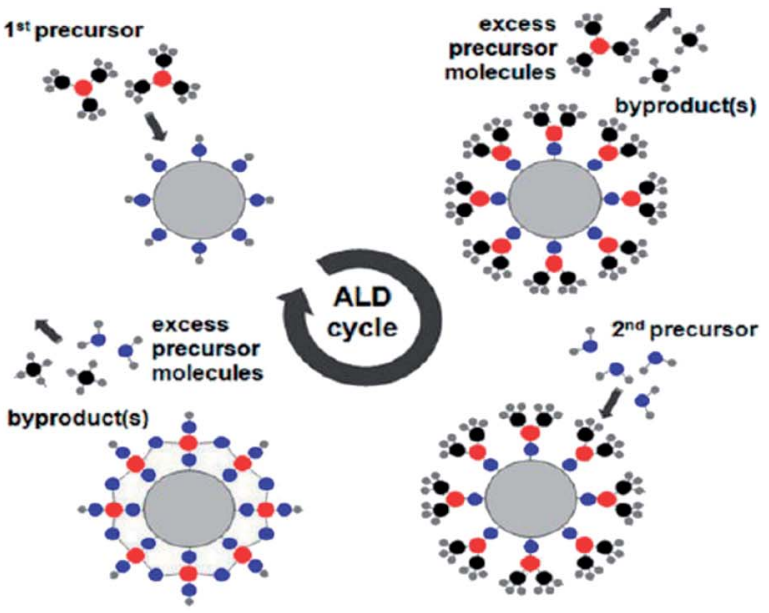

(a)

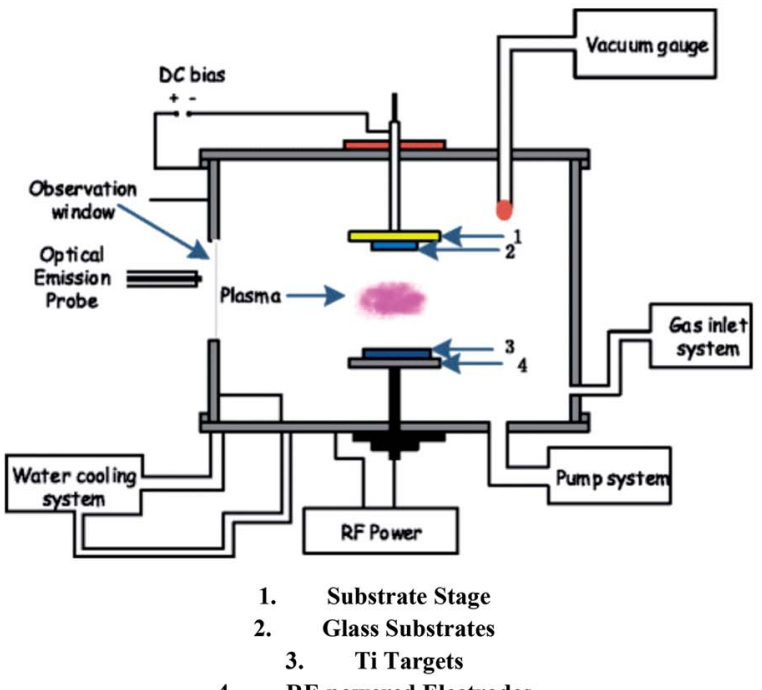

4. RF-powered Electrodes

(b)

Fig. 11 Schematic representation of various seeding methods: (a) $\mathrm{ALD}^{83}$ and (b) RF magnetron sputtering. ${ }^{84}$

the nanoparticles. ${ }^{93}$ The electromechanical conversion efficiency of the PENG is limited by polarization screening effects induced by free charge carriers inside the piezoelectric NSs. ${ }^{\mathbf{9 4}}$ In addition, the stability of the device is affected by continuous use owing to the loss of polarization. Wang et al. showed the transformation of electrospun PVDF nanofibers under the influence of an ultra-high electrical field from non-polarized $\alpha$ phase to polarized $\beta$-phase structures. Polarization and a piezoelectric effect are observed in the case of individual collagen fibrils. ${ }^{95-97}$ Collagen exhibits uniaxial polarization along the long axis of the collagen fibril owing to a change in the magnitude of the dipole moments. ${ }^{98}$ Kholkin et al. observed strong shear piezoelectric activity in self-assembled diphenylalanine peptide nanotubes (PNTs) and electric polarization, which was directed along the tube axis. ${ }^{\mathbf{9 9}}$ The polarization efficiency depends upon the values of the external electric field, the duration of the impact and the polarization temperature. ${ }^{\mathbf{1 0 0}}$

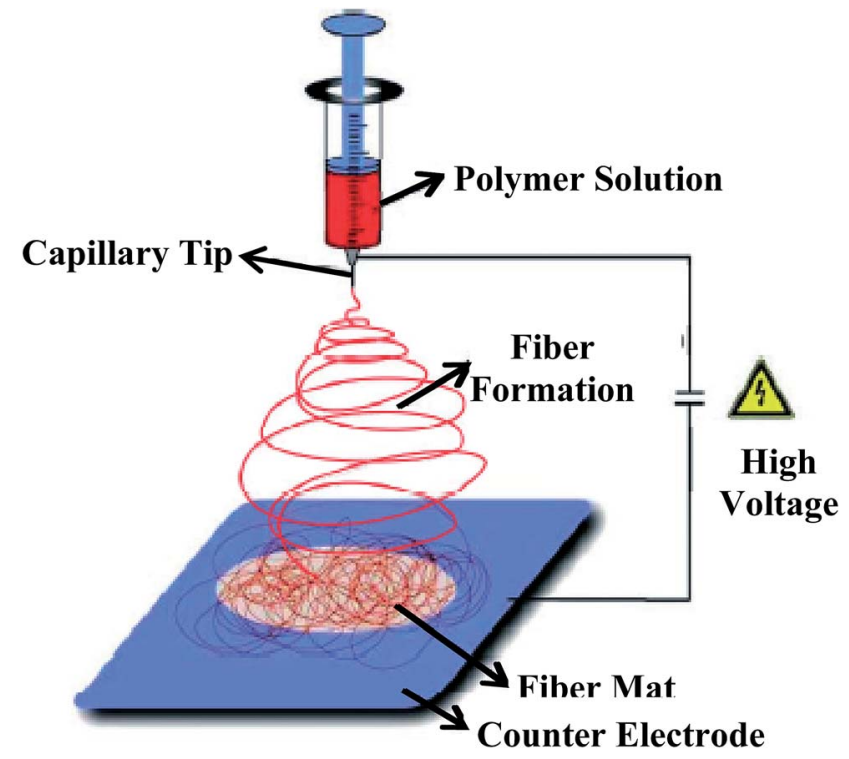

Fig. 12 Schematic illustration of a typical laboratory setup for conventional electrospinning with vertical feeding of the solution. ${ }^{90}$

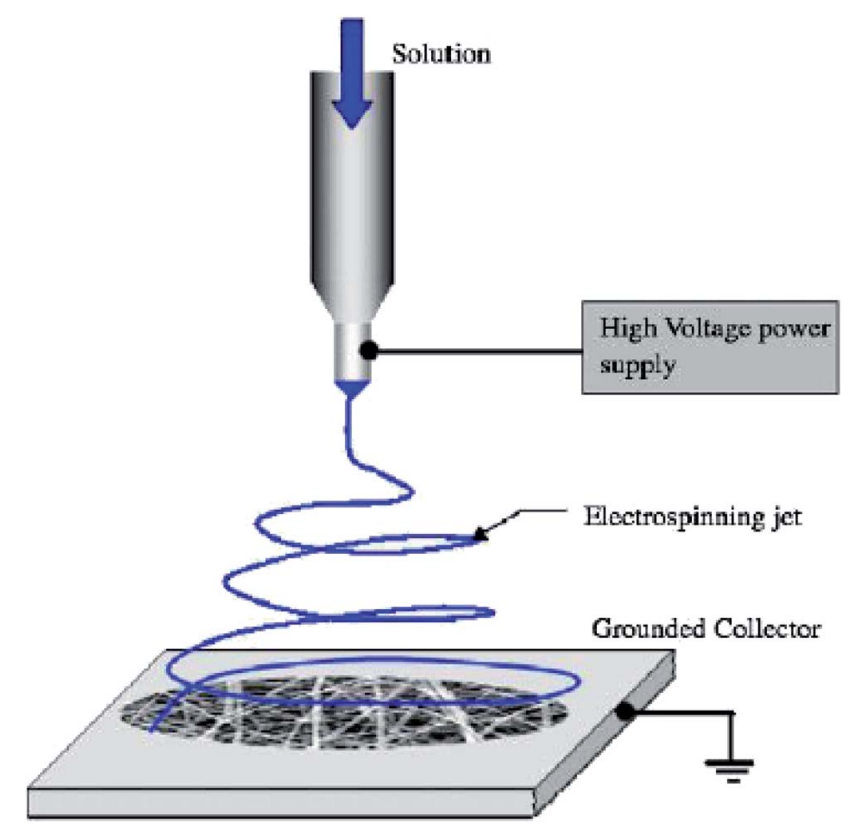

Fig. 13 Typical setup for electrospinning using a grounded collector. ${ }^{91}$

\section{Materials}

The mechanism of power generation mainly relies on the electromechanical properties of the material, as well as the formation of a Schottky contact between the tip of the NS and the metal electrode. Numerous studies have demonstrated novel nanodevices and the synthesis of new nanomaterials for enhancing the performance of NGs. Several inorganic materials, such as ZnO, ${ }^{\text {9,101,102 }}$ 1-D ZnO nanorods on a 2-D graphene electrode $^{\mathbf{1 0 3}}$ and $\mathrm{ZnS},{ }^{104}$ have been developed to design potential 


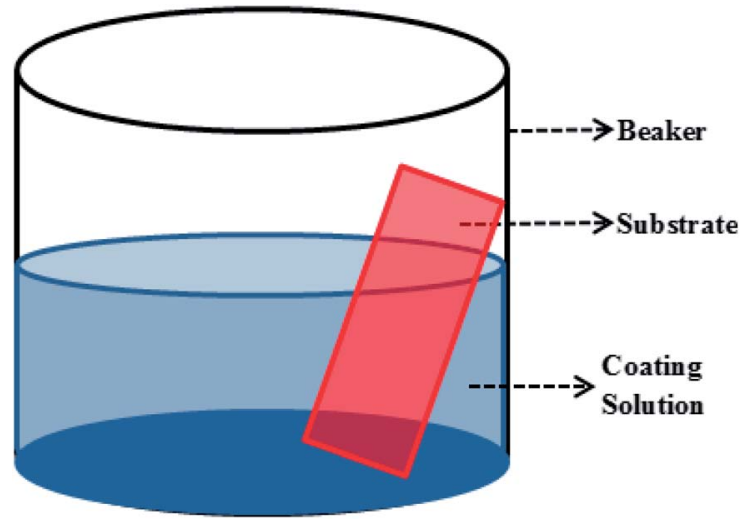

Fig. 14 Schematic of dip coating technique.

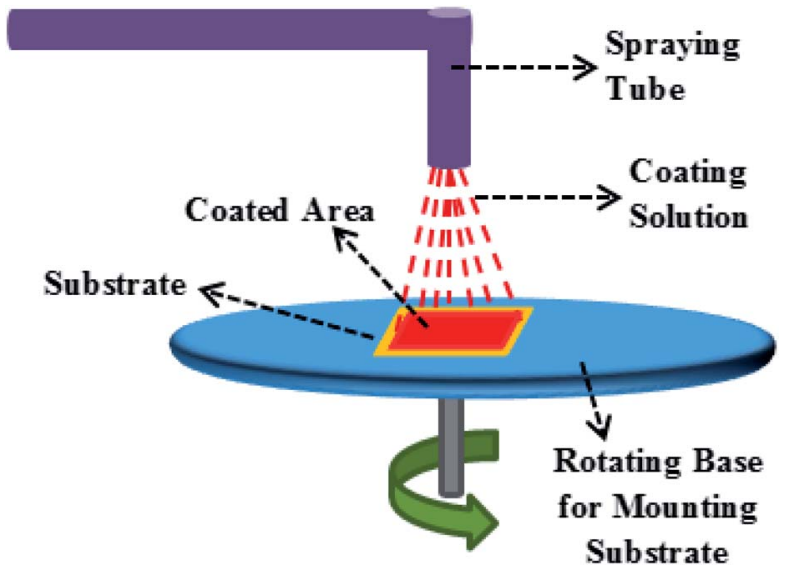

Fig. 15 Experimental setup used for spin coating on a substrate.

energy harvesters. Piezoelectric semiconductor materials with the wurtzite structure, such as $\mathrm{ZnO}, \mathrm{GaN}$, and $\mathrm{CdS}$, have been a focus for energy harvesting ${ }^{105-107}$ because they exhibit both semiconducting and piezoelectric properties simultaneously.

$\mathrm{ZnO}$ is a well-known suitable candidate with superior characteristics for the fabrication of NGs because of its high exciton binding energy, ideal wide band gap $(3.4 \mathrm{eV}),{ }^{9}$ biocompatibility, biosafety, ${ }^{108}$ environment-friendliness, abundant availability, chemical stability, ${ }^{109}$ high transparency ${ }^{110}$ relatively low cost, simple fabrication process ${ }^{111}$ and uniform growth on both conventional and unconventional substrates. ${ }^{112}$ It has highly diverse and abundant configurations of nanostructures, ${ }^{113}$ such as nanowires, ${ }^{114}$ nanorods, nanoflowers, nanosprings, ${ }^{115}$ nanobelts (NBs), ${ }^{116}$ nanobows, ${ }^{69}$ nanohelices, ${ }^{82}$ nanocombs, nanosheets and nanorings. ${ }^{117}$ One of the most surprising features of ZnO NWs is the coupling of semiconducting and piezoelectric (PZ) properties, which can form the basis for electromechanically coupled sensors and transducers. It can also be used for biomedical applications because of its minimal toxicity. A ZnO $\mathrm{NW}$ is able to generate AC and DC output in vertical and tilted alignments, respectively. A ZnO NW can easily be bent by an external force. ${ }^{111}$ Zhou et al. (2008) proposed $\mathrm{ZnO}$ nanostructures as promising candidates for use in mechanical/

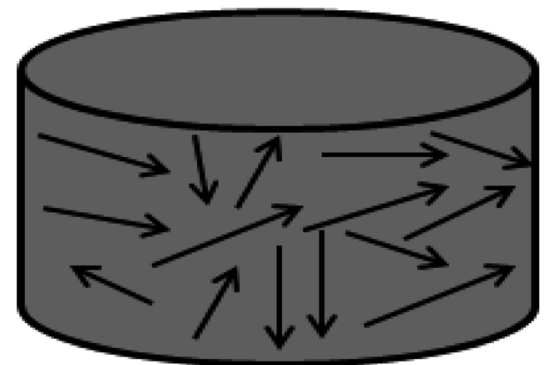

(a)

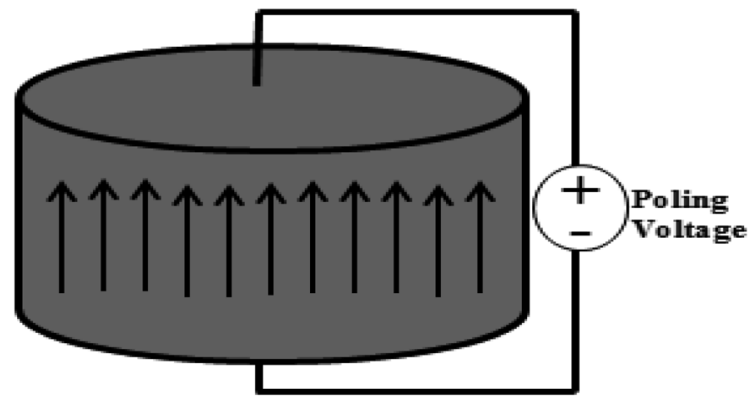

(b)

Fig. 16 (a) Before poling. (b) After poling

electrical energy conversion devices owing to their exceptional robust properties. ${ }^{118} \mathrm{ZnO}$, owing to its semiconducting and piezoelectric properties, is considered highly favorable in UV lasers, ${ }^{119}$ UV sensors, ${ }^{120}$ light-emitting diodes, ${ }^{121}$ gas sensors ${ }^{122,123}$ and solar cells. ${ }^{124}$ Lu et al. (2009) introduced a ptype $\mathrm{ZnO} \mathrm{NW}$ and proved experimentally that it was able to generate an output signal nearly 10 times that of an n-type ZnO $\mathrm{NW} .{ }^{3}$ Even though the enhanced performance of NGs based on $\mathrm{ZnO}$ has been well described, they generate relatively low output power because of the low piezoelectric coefficient of $\mathrm{ZnO}$ in comparison with $\mathrm{BaTiO}_{3}$ (ref. 125 and 126) and PZT, ${ }^{127}$ which remains the main problem. ${ }^{128,129}$

Inorganic materials have a limited number of applications, because of their low formability and brittle nature. Polymers are used as alternative materials in applications in which a lightweight generator design is required. ${ }^{130-132}$

Poly(vinylidene fluoride) (PVDF) and its copolymers are the most commonly studied polymers for piezoelectric generators. ${ }^{133-137}$ PVDF can be used to fabricate different structures such as PVDF nanofibers, ${ }^{18}$ single PVDF nanofibers, ${ }^{138}$ PVDF films, ${ }^{139}$ porous PVDF films, ${ }^{18,140}$ PVDF mats, ${ }^{141}$ PVDF microbelts, ${ }^{142}$ and PVDF nanofiber membranes. ${ }^{143}$ PVDF was also suggested for designing an NG by Chang et al. ${ }^{138}$ (2010). Fang et al. (2011) showed that the power-generating capability of electrical generators based on PVDF nanofibers was improved in comparison with that of thin PVDF films. ${ }^{143}$

Cadmium sulfide (CdS) is a wurtzite-type piezoelectric material with an energy band gap of about $2.5 \mathrm{eV},{ }^{144}$ which exhibits improved performance because of its coupled piezoelectric, optoelectric ${ }^{145}$ and semiconducting ${ }^{146}$ properties. Vertically grown cadmium sulfide (CdS) NW arrays were 
fabricated by Lin et al. in $2008 \cdot{ }^{\mathbf{1 4 7}} \mathrm{CdS}$ is used for the synthesis of various nanostructures such as NWs, ${ }^{148}$ nanobelts, ${ }^{149}$ nanocombs, ${ }^{63} \mathrm{NW}$ arrays ${ }^{150}$ nanotubes ${ }^{151}$ and tetrapods. ${ }^{64}$

$\mathrm{GaN}$ is well known for its excellent optoelectronic properties, with a wide direct band gap, high mobility, and excellent thermal stability. ${ }^{\mathbf{1 5 2 , 1 5 3}}$ GaN nanowire (NW) arrays distributed with threefold symmetry have been successfully grown on a sapphire substrate and were observed to be highly stable, with exceptional tolerance of atmospheric moisture. Huang et al. (2010) reported that n-type GaN NW arrays produced a negative piezoelectric output voltage of $20 \mathrm{mV}$, whereas $5-10 \%$ of the NWs exhibited increased piezoelectric voltages of about $100 \mathrm{mV} \cdot{ }^{154} \mathrm{GaN}$ nanowires (NWs) synthesized by a vapor-liquidsolid (VLS) process were assembled rationally by Lin et al. in 2011 and generated an output voltage of up to $1.2 \mathrm{~V}$ and an output current density of $0.16 \mu \mathrm{A} \mathrm{cm}^{-2} .^{155}$

Alpha-quartz was grown at the interface between the core and the shell of silicon nanowires by Yin et al. in $2013 .{ }^{156}$ These Si NWs containing alpha-quartz exhibited improved piezoelectric responses and could be used as starting materials for the fabrication of PENGs. When a free-falling object of $300 \mathrm{~g}$ was made to fall on the Si nanowires from a height of $30 \mathrm{~cm}$, the harvested output voltage was $36.5 \mathrm{~V}$ and the response current was $1.4 \mu \mathrm{A}$.

The overall performance of a piezoelectric nanogenerator relies on the piezoelectric constant of the piezoelectric material. Several types of research have been carried out to develop a material with enhanced piezoelectric properties.

Lead zirconate titanate (PZT) was found to provide the ideal solution and has become dominant among various piezoelectric materials ${ }^{\mathbf{1 2 7}}$ because of its superior piezoelectric and electromechanical characteristics. ${ }^{157} \mathrm{Wu}$ et al. (2001) fabricated a nanogenerator (NG) based on parallel PZT NWs, and a high output voltage and short-circuit current of $6 \mathrm{~V}$ and $45 \mathrm{nA}$, respectively, were generated. ${ }^{\mathbf{1 5 8}}$

Lead magnesium niobate-lead titanate (PMN-PT) is a piezoelectric material of which the piezoelectric response has been greatly improved by maintaining an ideal composition and orientation. In 2012, PMN-PT nanowires with a very high piezoelectric constant were fabricated by a hydrothermal approach ${ }^{\mathbf{1 5 9}}$ and were then assembled into an energy-harvesting device. ${ }^{\mathbf{1 6 0}}$ The piezoelectric constant was increased further by developing a single-crystal PMN-PT nanobelt. ${ }^{\mathbf{1 6 1}}$

PZT has been an irreplaceable piezoelectric material for a decade. However, the lead content in PZT is toxic and results in severe hazards to the environment. Hence, there is a great need for the development of a piezoelectric material that can replace the widespread use of lead-based materials, and therefore many researchers have started to focus on the development of lead-free piezoelectric materials. Lead-free piezoelectric materials such as $\mathrm{BaTiO}_{3}(\mathrm{BT}),{ }^{\mathbf{1 2 5 , 1 2 6}} \mathrm{NaNbO}_{3}(\mathrm{NN}),{ }^{15} \mathrm{KNbO}_{3}(\mathrm{KN})$ and BCTZ have been used for the synthesis of piezoelectric nanogenerators (PENGs).

Zhang et al. (2016) reported that a flexible $\mathrm{BaTiO}_{3}$ NG composed of a bacterial cellulose film can generate an output voltage of $1.5 \mathrm{~V}$ when it is subjected to bending. ${ }^{162}$ The output signal of a barium titanate $\left(\mathrm{BaTiO}_{3}\right) \mathrm{NW}$ was observed to be 16 times stronger than that of a similar $\mathrm{ZnO}$ nanowire, and it was reported that materials with the perovskite structure exhibit improved piezoelectric characteristics when compared with materials with the wurtzite structure. ${ }^{163}$ NGs have been fabricated and improved piezoelectric properties were observed from BT-based NSs such as BT nanoparticles, BT nanotubes, and BT films. ${ }^{\mathbf{1 2 5 , 1 6 4 - 1 6 6}}$ However, the applications of BT are limited by its low Curie temperature of $120^{\circ} \mathrm{C}$, so that it cannot be employed for the design of NGs that are operated at high temperatures.

Because the electrical energy produced by an NG is proportional to its piezoelectric constant, NWs of NN exhibit a relatively low output voltage and current owing to its extremely low piezoelectric strain constant $\left(d_{33}\right)$ of $50 \mathrm{pC} \mathrm{N}^{-1} \cdot \cdot^{15,131,167}$

Orthorhombic (OR) KN nanorods generated an extremely low current and voltage, ${ }^{\mathbf{1 6 8 - 1 7 0}}$ whereas $\mathrm{KN}$ nanowires with both tetragonal and OR structures exhibited an increased output voltage and current of $10.5 \mathrm{~V}$ and $1.31 \mathrm{~A}$, respectively. ${ }^{\mathbf{1 7 0}}$

Titanium dioxide $\left(\mathrm{TiO}_{2}\right)$ is an outstanding material and represents a most promising candidate in the fields of environmental purification, photoelectrochemical solar energy conversion and optical coating applications. ${ }^{171}$ These noteworthy applications are due to its many improved characteristics such as chemical stability, non-toxicity, ${ }^{172}$ strongly oxidizing photogenerated holes, exceptional energy conversion ability, transparency, wide band gap (3.1 eV) and relatively high refractive index $(n=2.6) .{ }^{173}$ Thin $\mathrm{TiO}_{2}$ films are used as gate insulators for metal oxide semiconductor (MOS) device applications, and the leakage current has been reduced owing to its high dielectric constant. ${ }^{174}$ Kajitvichyanukul et al. (2005) fabricated thin $\mathrm{TiO}_{2}$ films on a glass substrate by means of a sol-gel technique and dip coating. ${ }^{175 a}$ The synthesized thin $\mathrm{TiO}_{2}$ films displayed improved photoactivity and efficiency in comparison with those of $\mathrm{TiO}_{2}$ powder. Andrej et al. (2017) fabricated nanostructured titanium dioxide $\left(\mathrm{TiO}_{2}\right)$ using a simplified solgel procedure from the organometallic precursor titanium tetraisopropoxide (TTIP) ${ }^{175 b}$ and performed thermal analysis.

Sodium potassium niobate $(\mathrm{NKN})$ is a suitable piezoelectric ceramic material for replacing lead-based piezoelectric materials, ${ }^{176-179}$ with good piezoelectric properties and a high Curie temperature. ${ }^{\mathbf{1 8 0 - 1 8 2}}$ NKN-based nanomaterials such as nanoparticles, nanofibers, and thin films have also been developed for energy-harvesting applications. ${ }^{183-187}$ However, NKN-based NGs have limited use because of their poor electrical performance. ${ }^{\mathbf{1 8 3 - 1 8 6}}$

The lead-free material BCTZ is considered to be one of the most promising alternatives to traditional lead-based piezoelectric materials owing to its excellent coupling of piezoelectric and ferroelectric properties ${ }^{87,188-192}$ and its high piezoelectric constant of $620 \mathrm{pC} \mathrm{N}^{-1}{ }^{193}$ Yanfei et al. (2017) proposed a biocompatible PENG based on lead-free BCTZ nanorods using an electrospinning method. ${ }^{194}$ An open-circuit voltage and a short-circuit current of $\sim 0.8 \mathrm{~V}$ and $\sim 7 \mathrm{nA}$, respectively, were observed upon tapping the NG.

Jong et al. (2011) synthesized an efficient flexible lead-free $\mathrm{NaNbO}_{3}$-based piezoelectric nanowire with a PDMS polymer composite and Au/Cr-coated polymer films using a hydrothermal method at a moderate temperature. ${ }^{15}$ When a compressive strain of $0.23 \%$ was applied across the NW, an 
output voltage of $3.2 \mathrm{~V}$, an output current of $72 \mathrm{nA}$ and a current density of $16 \mathrm{nA} \mathrm{cm}^{-2}$ were generated.

Zinc stannate $\left(\mathrm{ZnSnO}_{3}\right)$ is a novel material for research owing to its outstanding coupling of piezoelectric, pyroelectric and ferroelectric properties with relatively high electric and structural ordering temperatures of up to $700{ }^{\circ} \mathrm{C} .{ }^{195,196}$ It is a significant material belonging to the ternary metal oxide group. It has been exclusively used in the field of gas sensing because of its attractive optical properties, exceptional electronic properties ${ }^{197,198}$ and excellent chemical response. ${ }^{199}$ Lee et al. (2014) reported that the physical properties of $\mathrm{ZnSnO}_{3}$ can be maintained by varying its morphology, crystallinity, dimensions, and fabrication techniques. ${ }^{200}$ The gas-sensing capabilities of $\mathrm{ZnSnO}_{3}$ nanostructures can be improved by synthesizing hollow structures with minimal gas diffusion lengths and a greater specific surface area than other nanomaterials. ${ }^{201,202} \mathrm{ZnSnO}_{3}$ has a high piezoelectric coefficient of approximately $59 \mu \mathrm{C} \mathrm{cm}^{-2}$, which is almost 12 times that of $\mathrm{ZnO}$ (approximately $5 \mu \mathrm{C} \mathrm{cm}^{-2}$ ).

\section{Optimization}

Therefore, optimization has to be performed to maximize the conversion efficiency of a nanogenerator. Optimization of the design of NGs has been carried out in two ways: either by increasing the parameters that lead to enhancements in piezoelectric response or by diminishing the factors that are responsible for the degradation of device performance.

Lin et al. (2007) found that the reaction time had to be adjusted so that tellurium (Te) with the desired aspect ratio could be developed. ${ }^{85}$

Ahmad et al. (2017) grew $50 \mathrm{~nm} \mathrm{ZnO}$ nanowires on an indium tin oxide (ITO)-coated polyethylene terephthalate (PET) substrate. ${ }^{70}$ The annealed substrate was immersed in a nutrient solution of HMTA and zinc nitrate hexahydrate for two hours. It was found that the density and diameter of the NWs were controlled by maintaining the concentration of the nutrient solution and the growth time, respectively. It was also observed that the piezoelectric potential can be increased by reducing the diameter of the NWs. The scattering phenomenon within the NWs can be enhanced by reducing the diameter, which further reduces the reverse leakage current through the NWs. Hence, a piezoelectric response is triggered.

The piezoelectric properties of a material can be improved by means of doping. Suyitno et al. (2014) proposed that doping ZnO-based nanofibers with cobalt promotes the growth of the fibers, as well as increasing the voltage generated by the NG. ${ }^{203}$ Kanjwal et al. (2011) stated that the doping of nanofibers with cobalt increases the elastic modulus of the fibers. ${ }^{204}$ The value of $d_{33}$ has been increased owing to the addition of aluminium to ZnO fibers. Subagiyo et al. (2017) reported that extensive improvements to crystal properties are also made possible by means of co-doping; for example, the addition of aluminum and cobalt in a $75: 25$ ratio to $\mathrm{ZnO}$ nanofibers led to an increase in the value of $d_{33}$ from -3.9 to $-4.1 \mathrm{pC} \mathrm{N}^{-1}$. 205

The performance of a generator can also be enhanced by controlling the temperature. Zhu et al. (2005) reported that the piezoelectric properties of $\mathrm{ZnO}$ fibers improved with an increase in the sintering temperature. ${ }^{109}$ It was found that the value of $d_{33}$ of $\mathrm{ZnO}$ fibers was drastically reduced when the sintering temperature was raised above $500{ }^{\circ} \mathrm{C}$. NKN nanorods that were annealed at $450{ }^{\circ} \mathrm{C}$ had several $\mathrm{OH}$ and $\mathrm{H}_{2} \mathrm{O}$ defects and an unstable $\mathrm{RH}$ structure. As the annealing temperature was increased, these defects disappeared from the NKN nanorods. ${ }^{206}$

Hyeongwook et al. (2016) developed a nanowire using carbon nanotube aerogel sheet electrodes. ${ }^{207}$ The reverse leakage current through the nanowire was greatly reduced by reducing the diameter so that an increase in output voltage was achieved. The piezoelectric potential could be developed and controlled by means of the Schottky contact that was formed between the metal electrode and the tip of the NS.

Zafar et al. (2017) fabricated a ZnO-based NG and observed that the durability and performance of the generator could be improved by the addition of a host layer. ${ }^{69}$ The generated power could be maximized by increasing the size of the NG. The addition of the host layer and the increase in the size of the generator made the device expensive. Hence, the trade-off should be carefully maintained between the output performance and the cost of an NG. Chiang et al. (2012) fabricated a $\mathrm{CuO}$ film and found that the conductivity of the film could be increased by two orders of magnitude by replacing $\mathrm{Cu}^{2+}$ ions with $\mathrm{Li}^{+}$ions of the same radius. ${ }^{208}$

Several works have presented improvements in the electrical characteristics of PVDF-based NGs by inducing the formation of the $\beta$-polymorph in PVDF films via the inclusion of graphene. ${ }^{209-215}$ Abolhasani et al. (2017) found that the addition of the desired amount of graphene to reinforced PVDF composite nanofibers led to a substantial increase in the open-circuit voltage of the nanofibers. ${ }^{86}$ It was experimentally observed that an increase in the graphene content to above $0.1 \mathrm{wt} \%$ led to a decline in the voltage of randomly oriented nanofibers. Xue et al. (2012) produced reduced graphene oxide (rGO)-PVDF nanocomposites with an increased open-circuit voltage of $4 \mathrm{~V}$ with $0.2 \mathrm{wt} \%$ graphene. ${ }^{210}$

Haibo et al. (2017) found that polymorphic-phase (PP) NKN nanorods exhibited more directions for dipole rotation than orthorhombic (OR) and rhombohedral (RH) NKN nanorods grown on the same $\mathrm{Nb}^{5+}$-doped $\mathrm{SrTiO}_{3}$ substrate. ${ }^{206} \mathrm{PP}$ NKN nanorods of $0.7 \mathrm{~g}$ possessed a high piezoelectric strain constant of $175 \mathrm{pm} \mathrm{V}^{-1}$ and generated a high open-circuit voltage of $35 \mathrm{~V}$ and a short-circuit current of 5.01 A. They harvested a maximum power of $16.51 \mathrm{~W}$ across a resistive load of $10.0 \mathrm{M} \Omega$.

The piezoelectric potentials generated at the tips of nanowires will be balanced by the migration of free carrier electrons in the nanowires. Hu et al. (2009) suggested that surface desorption and native defects are the major reasons for the formation of free carriers. ${ }^{216} \mathrm{Lu}$ et al. (2015) proposed that these free electrons lead to the degradation of piezoelectric performance, ${ }^{217}$ which is referred to as the screening effect.

The effect of screening electrons can be reduced by improving the intrinsic properties of NGs using surface passivation, oxygen plasma and thermal annealing. ${ }^{218-222} \mathrm{Hu}$ et al. (2012) enhanced the performance of a single-layer VING with a maximum output voltage of up to $20 \mathrm{~V}$ and an output 
current of up to $6 \mu \mathrm{A}$ by treating $\mathrm{ZnO}$ nanowires with oxygen plasma or annealing them in air. ${ }^{218}$

The output power of PENGs is limited owing to the piezoelectric screening effect caused by high electron concentrations in $\mathrm{ZnO}$ nanostructures. This drawback has been rectified by introducing several p-type materials such as p-ZnO, p-CuO, P3HT, ZnS, NiO, PEDOT:PSS and p-Si to form a p-n junction. ${ }^{\text {104,223-230 }}$ Unfortunately, user-friendly p-type polymers are expensive. Another approach for suppressing the screening effect comprises the addition of a p-type Li-doped $\mathrm{Cu}_{2} \mathrm{O}$ (LCO) layer, which improves the piezoelectric properties by reducing the total capacitance. Cho et al. (2017) demonstrated that a PENG with a ptype LCO layer generated relatively high power of up to $\sim 52 \mu \mathrm{W} .{ }^{81}$ Lu et al. (2016) reported that the screening effect can also be effectively reduced by forming a Schottky contact via the addition of gold (Au) particles to a ZnO surface. ${ }^{231}$ Hence, the performance was enhanced owing to reductions in the carrier concentration and capacitance, which gave rise to a voltage and current density of $2 \mathrm{~V}$ and $1 \mathrm{~A} \mathrm{~cm}^{-2}$, respectively.

Zhu et al. (2012) reported that a polymethyl methacrylate (PMMA) layer added at the interface between $\mathrm{ZnO}$ and the top metal electrode acts as an insulating layer that blocks the movement of electrons across the interface ${ }^{\mathbf{1 0 6}}$ and leads to a reduction in the screening effect by avoiding the leakage of current.

The screening effect can also be reduced by varying the geometry of the device. Zho et al. (2012) presented a novel design topology for a ZnO VING, and the thickness of the PMMA layer between the top of the nanowires and the top electrodes was reduced to form an infinite potential barrier. ${ }^{\mathbf{1 0 6}}$ This innovative design was able to generate an output voltage of $58 \mathrm{~V}$ and an output current of $134 \mu \mathrm{A}$.

Pham et al. (2013) stated that the output performance of $\mathrm{ZnO}$ nanorods can also be enhanced by surface passivation using ultraviolet light. ${ }^{219}$ Lin et al. (2008) improved the efficiency of CdS using stimulation by white light. ${ }^{232}$

Pradel et al. (2013) reported that the length of ZnO NRs could be further increased by adding ammonia to the reaction solution, and the longer $\mathrm{ZnO}$ NRs that were prepared were demonstrated to have a higher piezopotential. ${ }^{233}$ The construction of a fiber-based three-dimensional hybrid nanogenerator (FBHNG) using a foldable polymer film with sputtered copper electrodes on both sides is a feasible way to enhance the output of the FBHNG.

The electrodes used in an NG can also determine the efficiency of the device. Hence, proper care has to be taken when choosing the electrode materials. Metals are not extensively used as electrodes in the design of NGs because of their reduced flexibility and the complicated procedures used for threedimensional microstructural patterning of metals. ${ }^{234-236}$ Polydimethylsiloxane (PDMS)-CNT is used as the bottom electrode because it provides extreme flexibility and makes the harvesting element relatively stretchable in order to make the NG device fully stretchable. ${ }^{237-239}$ Graphene exhibits high thermal conductivity and provides a fast temperature gradient for the NG. Hence, graphene acts as a suitable candidate for the electrode. ${ }^{\mathbf{2 4 0 , 2 4 1}} \mathrm{ZnO} \mathrm{NRs}$ were grown radially around carbon fibers

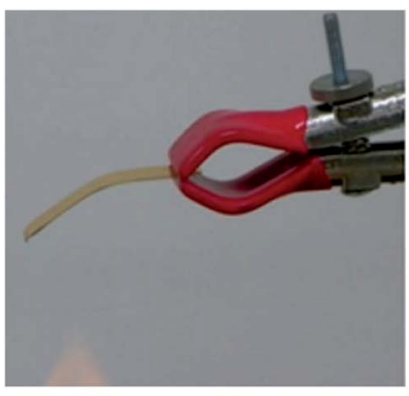

(a)

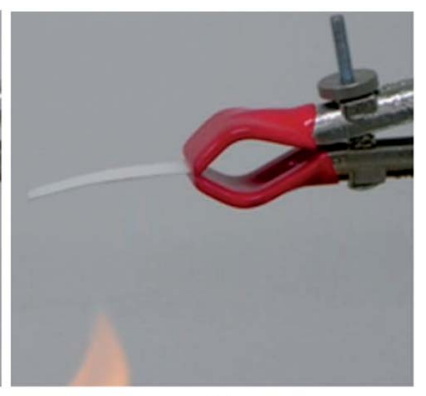

(b)
Fig. 17 Thermal stability of (a) paper-based nanogenerator and (b) polymer-based nanogenerator. ${ }^{243}$

and thus formed an all-round electrode that could utilize all the ZnO NRs on the fibers, so that the contact area and electrical output were increased. ${ }^{242}$ A flexible polymer film can be folded at any angle and is a perfect candidate for an all-round electrode.

The applications of nanogenerators are limited because of their temperature instability, even though they exhibit increased piezoelectric potentials owing to a drastic increase in thermally induced stress on polymer substrates due to the large difference in the coefficient of thermal expansion (CTE) between inorganic films and polymer substrates at high temperatures. Jeong et al. (2014) developed a flexible piezoelectric thin film on plastic substrates instead of bulk sapphire substrates using a laser lift-off (LLO) process, which was able to generate $140 \mathrm{~V}$ with an output current of $10 \mu \mathrm{A} \cdot{ }^{244} \mathrm{Kim}$ et al. (2011) reported a foldable PENG with high thermal stability using cellulose paper as a substrate, which overcame the unstable electrical response of plastic-based PENGs due to thermally induced stress. ${ }^{243}$ Cellulose paper is a suitable substrate owing to its flexibility, thermal stability, light weight, abundant availability and low cost. An analysis of the thermal stability of a polymer-based PENG and a paper-based PENG is shown in Fig. 17. The paper-based PENG remained stable, whereas the polymer-based PENG underwent great variations in shape when subjected to heating for $1 \mathrm{~min}$ by an alcohol lamp.

Optimization has also been carried out in order to increase the current generated by PENGs in order to meet the needs of consumer electronics. The generation of current can be increased by controlling the growth and density of nanostructures. Uniformity of nanostructures is maintained by growing a regular array of nanowires with a uniform size and height. All the nanowires can be utilized for power generation by matching the nanowire pattern with the pattern on the electrode, so that the device can generate more current and its stability is improved.

\section{Hybrid energy harvesting}

The design of hybrid energy harvesters is a booming technology, which can enable ubiquitous power generation with excellent adaptability and increased power conversion efficiency by combining two power generation mechanisms. ${ }^{245} \mathrm{~A}$ 
hybrid harvester can be designed by integrating a PENG with other types of energy harvester, by combining different fabrication techniques, or by aggregating two piezoelectric materials. A fundamental analysis has to be performed when deciding the shape of a hybrid NG and the fabrication procedure used to combine different energy-harvesting mechanisms.

Li et al. (2014) designed an FBHNG that comprised a PENG and a TENG. ${ }^{242}$ The PENG was used to increase the efficiency of mechanical energy collection whenever the TENG was unable to harvest energy. Carbon fibers were used as an effective substrate for the design of an FBHNG owing to their superior properties such as light weight, cheapness, high conductivity and easy foldability. ${ }^{6,246}$ The twelve contact array points used for the TENG and PENG provided an output power density of $42.6 \mathrm{~mW}$ $\mathrm{m}^{-2}$ and $10.2 \mathrm{~mW} \mathrm{~m}^{-2}$, respectively. ${ }^{242}$

Zhang et al. (2015) developed an innovative approach to the design of a hybrid energy harvester by using two fabrication procedures to increase the mechanical reliability of a PENG. ${ }^{247}$ Kevlar microfiber-ZnO nanowires (NWs) were synthesized using surface coating and plasma etching techniques and displayed enhanced performance such as improved flexibility, robustness, and durability. The open-circuit voltage and shortcircuit current of the fabricated Kevlar microfiber-ZnO NWs were $1.8 \mathrm{mV}$ and $4.8 \mathrm{pA}$, respectively.

Gupta et al. (2014) designed a hybrid stable PENG with exceptional power generation capacity by using singlecrystalline piezoelectric perovskite zinc stannate $\left(\mathrm{ZnSnO}_{3}\right)$ composite nanocubes and PDMS without electrical poling

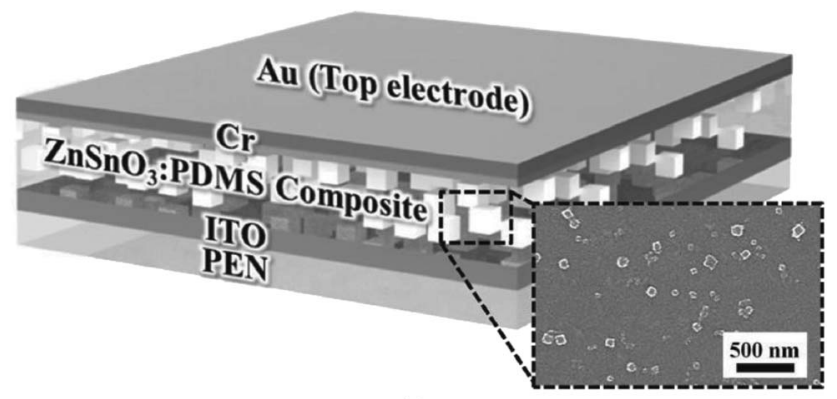

(a)

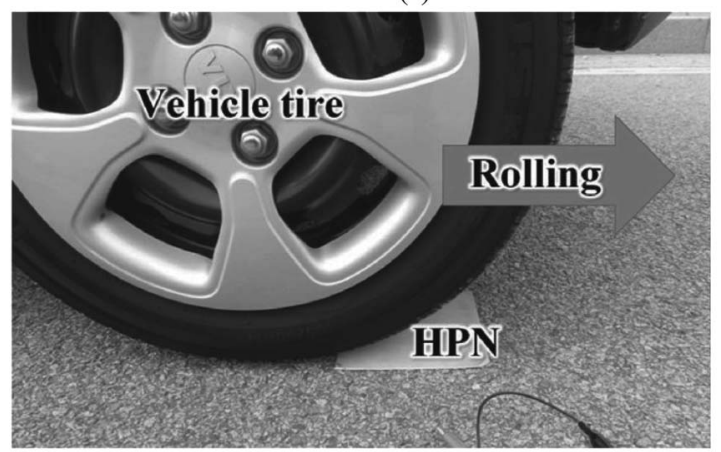

(b)

Fig. 18 (a) Schematic diagram of $\mathrm{ZnSnO}_{3}$ :PDMS-based flexible hybrid PENG. (b) Experimental setup used for the generation of power from the hybrid PENG under a rolling vehicle tire. ${ }^{200}$ treatment. ${ }^{200}$ A schematic diagram of the hybrid PENG is shown in Fig. 18(a). The hybrid $\mathrm{ZnSnO}_{3} / \mathrm{PDMS}$-based PENG that was developed harvested more power when subjected to vertical compression than when it underwent bending and folding. This hybrid NG exhibited unique characteristics such as mechanical durability, excellent robustness, and largely unidirectional power generation behavior. When a vehicle tire was rolled over a fabricated single hybrid NG cell based on $\mathrm{ZnSnO}_{3} / \mathrm{PDMS}$, as shown in Fig. 18(b), an output voltage of about $20 \mathrm{~V}$ and an output current density of about $1 \mu \mathrm{A} \mathrm{cm}^{-2}$ were successfully obtained.

Gao et al. (2015) proposed a hybrid piezoelectric fiber composed of aligned $\mathrm{BaTiO}_{3}$ nanowires and PVC polymer. ${ }^{248}$ The $\mathrm{BaTiO}_{3}$ nanowires acted as active materials and improved the piezoelectric properties, and the flexibility of the fiber was enhanced by means of the PVC polymer. This efficient flexible fiber could be woven into fabric together with cotton and interdigitated electrodes. An LCD could also be powered up by generating power from this fabric nanogenerator on an elbow pad, which was capable of generating an output voltage of $1.9 \mathrm{~V}$ and an output current of $24 \mathrm{nA}$.

Alluri et al. (2015) developed a flexible hybrid PENG made of a hybrid film composed of highly crystalline BTZO nanocubes embedded in a PVDF polymer matrix solution. ${ }^{249}$ The fabricated BTZO/PVDF film paved the way for the development of eco-friendly devices, active sensors, and efficient flexible NGs. BTO/PVDF generated a voltage of $7.99 \mathrm{~V}$ with an output current of $1.01 \mu \mathrm{A}$, whereas the proposed flexible hybrid film exhibited improved electrical performance with values of up to $\sim 11.9 \mathrm{~V}$ and $\sim 1.35 \mu \mathrm{A}$, respectively, under the same force of $11 \mathrm{~N}$. The durability and reproducibility of the hybrid PENG were also shown to be effective by performing stability testing.

Alam et al. (2015) improved the performance of a $\mathrm{ZnSnO}_{3} /$ PDMS-based hybrid PENG with multiwalled carbon nanotubes (MWCNTs) as a supplementary filling material. ${ }^{250}$ The electrical performance of the fabricated PENG was improved owing to the good distribution of $\mathrm{ZnSnO}_{3}$ nanocubes, and it generated an open-circuit voltage of $40 \mathrm{~V}$, a short-circuit current of $0.4 \mu \mathrm{A}$ and a power density of $10.8 \mu \mathrm{W} \mathrm{cm}{ }^{-3}$ without electrical poling.

Efficient energy harvesting from the ambient environment is possible using a hybrid device. There is an increasing demand for hybrid nanoscale harvesting devices that utilize two or more energy sources in order to harvest ubiquitous forms of energy, so that they can meet current requirements for energy.

Keun et al. (2014) presented an effective way of improving device performance with reductions in size and complexity so that a single NG can harvest two different kinds of energy. ${ }^{251}$ It was designed by the integration of piezoelectric and pyroelectric harvesting mechanisms. A hybrid energy-scavenging NG based on a micropatterned piezoelectric $\mathrm{P}(\mathrm{VDF}-\mathrm{TrFE})$ polymer, a micropatterned PDMS-CNT composite and graphene nanosheets was fabricated and was found to be relatively stretchable, robust and stable even after being subjected to multiple stretching and release cycles.

Wang et al. (2016) developed a flexible hybrid triboelectric and piezoelectric nanogenerator (TPENG) based on P(VDFTrFE) nanofibers and a thin PDMS/MWCNT composite with 
the combined effects of triboelectric and piezoelectric energy harvesters. ${ }^{252}$ The $\mathrm{P}(\mathrm{VDF}-\mathrm{TrFE})$ nanofibers performed the dual role of a piezoelectric functional layer and a triboelectric frictional layer. Doping with MWCNT improved the performance of the triboelectric nanogenerator. When the TPENG was subjected to a force of $5 \mathrm{~N}$, the peak-to-peak output voltage, output power and power density were observed to be $25 \mathrm{~V}, 98.56 \mu \mathrm{W}$ and $1.98 \mathrm{~mW} \mathrm{~cm}^{-3}$, respectively, during triboelectric operation, whereas values of $2.5 \mathrm{~V}, 9.74 \mu \mathrm{W}$, and $0.689 \mathrm{~mW} \mathrm{~cm}{ }^{-3}$ were obtained for piezoelectric operation. The proposed hybrid NG was found to be biocompatible, less expensive, lightweight, and flexible and can be easily fabricated by a low-cost MEMS process.

Park et al. (2013) integrated a silicon nanopillar solar cell with a PVDF NG using a spinning method, and a tandem NG was proposed. ${ }^{139}$ The device provided ubiquitous power by harvesting electrical power from both sound waves and solar energy. It could harvest a peak-to-peak output voltage of $0.8 \mathrm{~V}$ using $100 \mathrm{~dB}$ sound waves and exhibited a solar conversion efficiency of $3.29 \%$.

Han et al. (2013) presented an innovative three-dimensional rshaped hybrid NG design based on piezoelectric and triboelectric energy harvesting. ${ }^{253}$ The output performance of the device was enhanced by fabricating micro- or nanoscale devices on a PDMS surface, which was placed under an aluminum electrode on PVDF. The Al electrode was shared in common by both the piezoelectric and the triboelectric component. The piezoelectric and triboelectric generators exhibited an increased power density of $10.95 \mathrm{~mW} \mathrm{~cm}^{-3}$ and $2.04 \mathrm{~mW} \mathrm{~cm}^{-3}$, respectively. The hybrid r-shaped design exhibited relatively high reliability, as its performance was not degraded over 6000 continuous cycles under an external force with a frequency of $10 \mathrm{~Hz}$.

Yunlong et al. (2015) developed a triboelectric-pyroelectricpiezoelectric hybrid cell for high-efficiency energy harvesting during sliding motion and self-powered sensing. ${ }^{254}$ A TENG with a size of $63.5 \mathrm{~cm}^{2}$ generated a maximum power density of 146.2 $\mathrm{mW} \mathrm{m} \mathrm{m}^{-2}$ at a sliding frequency of $4.41 \mathrm{~Hz}$. The efficiency of mechanical energy harvesting has been increased to $26.2 \%$ by combining a TENG with a pyroelectric-piezoelectric nanogenerator (PPENG). The generated output was able to light up an LED directly with a prolonged illumination time and to charge a supercapacitor at twice the charging rate of a TENG.

\section{Application areas}

Because abundant available vibrations occur in our surrounding environment, piezoelectric energy harvesting is a promising solution to the limitations and inconveniences of the power supply delivered to current systems. NGs also overcome the limitations of size and complexity and facilitate the development of many self-powered devices. The performance of nanoscale energy-harvesting devices has been improved by various techniques, which have been discussed earlier. PENGs become an efficient alternative power supply and act as a driving force for various smart applications. This emerging technology acts as a stepping stone for the development of a green environment. Nanoscale piezoelectric harvesting devices have entered into use in various areas such as industrial manufacturing, automobiles, medicine, information, telecommunications, and so on.

\section{A. Self-powered nano/microsensors}

The miniaturized size of nanogenerators makes them possible power sources for low-power micro/nanoscale devices fixed in a location where mechanical vibrations are continuously available. Self-powered gas sensors with high stability and sensitivity have been obtained by means of one-dimensional metal oxide nanostructures such as $\mathrm{ZnO}, \mathrm{SnO}_{2}$ and $\mathrm{In}_{2} \mathrm{O}_{3}$ because of their superior properties such as a high surface-to-volume ratio. ${ }^{255-258}$

Deng et al. (2014) used a ZnO nanowire for the design of a self-powered active biosensor. ${ }^{259}$ The power generated from the nanowire acts as a power source as well as a biosensing signal. The output voltage of the nanowire under compressive deformation decreased gradually from $0.20370 \pm 0.0176 \mathrm{~V}$ (without IgG) to $0.03870 \pm 0.0035 \mathrm{~V}$ (with $10^{-3} \mathrm{~g} \mathrm{~mL}^{-1} \mathrm{IgG}$ ). Surface molecular adsorption can vary the density of free carriers on $\mathrm{ZnO}$ NWs, so that the screening effect can be regulated. Because ZnO NWs have the coupled properties of piezotronic and biosensing characteristics, this NW acts as a viable solution for the active detection of biomolecules.

Nie et al. (2014) developed a self-powered active gas sensor for $\mathrm{H}_{2} \mathrm{~S}$ based on a $\mathrm{CuO} / \mathrm{ZnO}$ nanoarray, in which the piezoelectric response acted as a power source as well as a sensing signal. ${ }^{260}$ At room temperature, the piezoelectric output of the nanoarray decreased relatively from $0.738 \mathrm{~V}$ to $0.101 \mathrm{~V}$ when the device was exposed to $800 \mathrm{ppm}_{2} \mathrm{~S}$. When the device was exposed to $\mathrm{H}_{2} \mathrm{~S}$ gas, the $\mathrm{CuO} / \mathrm{ZnO} \mathrm{p}-\mathrm{n}$ junction was modified to form a $\mathrm{CuS} / \mathrm{ZnO}$ ohmic contact. The electron density in the NW increased, which resulted in an additional screening effect.

Xue et al. (2013) fabricated an unpackaged $\mathrm{ZnO}$ nanowire (NW) PENG, which functioned as a self-powered active gas sensor even at room temperature because of the combination of the piezoelectric and gas-sensing characteristics of ZnO NWs. ${ }^{261}$ It seemed to exhibit exceptional sensitivity even in spite of the electron screening effect. ZnO NWs exhibit excellent performance when integrated with various devices such as solar cells, ${ }^{262}$ gas sensors, ${ }^{263}$ UV lasers, ${ }^{264}$ light-emitting diodes ${ }^{265}$ and photodetectors. ${ }^{266,267}$

A small PENG-based self-powered sensor provided high durability and could be operated even at a high fluid operating temperature of up to $120^{\circ} \mathrm{C}$ and over wide pressure ranges. ${ }^{249}$ Such sensors were successfully fabricated and used to measure water velocities at an outlet pipe.

\section{B. Self-powered electronics}

With the rapid growth of electronics, smart consumer electronics have become an essential item for comfortable human life. To increase the lifespan and power consumption of such electronic devices, wasted mechanical energy derived from finger movements as well as the tapping of touch screens by fingers has to be utilized by means of PENGs. Hence, nanoscale harvesting devices act as suitable power sources and bases for the development of self-powered devices. 
A PVDF/graphene-based nanogenerator has the ability to fully synchronize finger movements, and the generated energy can be used to power a commercial LED for 30 seconds. ${ }^{86}$ This PVDF generator has the ability to be used as a self-charging power source for powering portable personal electronic devices that require less power.

The stable output from the three-dimensional $r$-shaped hybrid NG could be used to charge capacitors, which act as power sources for microelectronic devices. ${ }^{253}$ The flat and adjustable substrate endows the device with exceptional integrability. The hybrid PENG could be integrated into the space button of a keyboard by using the plastic film of the keyboard as a frictional substrate. With gentle finger typing, a peak voltage of about $20 \mathrm{~V}$ was generated.

Hu et al. (2016) proposed an innovative anti-peep transparent PENG that was able to harvest energy from light finger tapping, and an output current of $0.8 \mathrm{nA}$ was generated. ${ }^{5}$ This transparent NG exhibited improvements in light transmittance and electromechanical properties upon aligning PZT nanowires on PDMS.

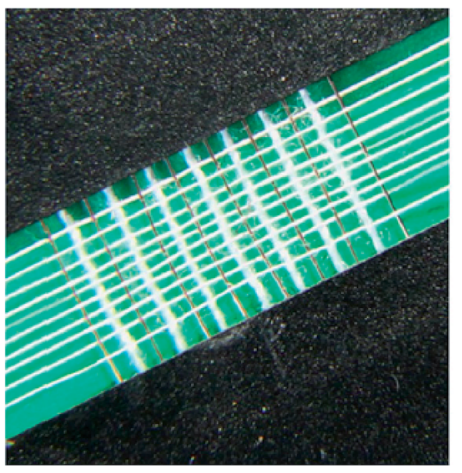

(a)

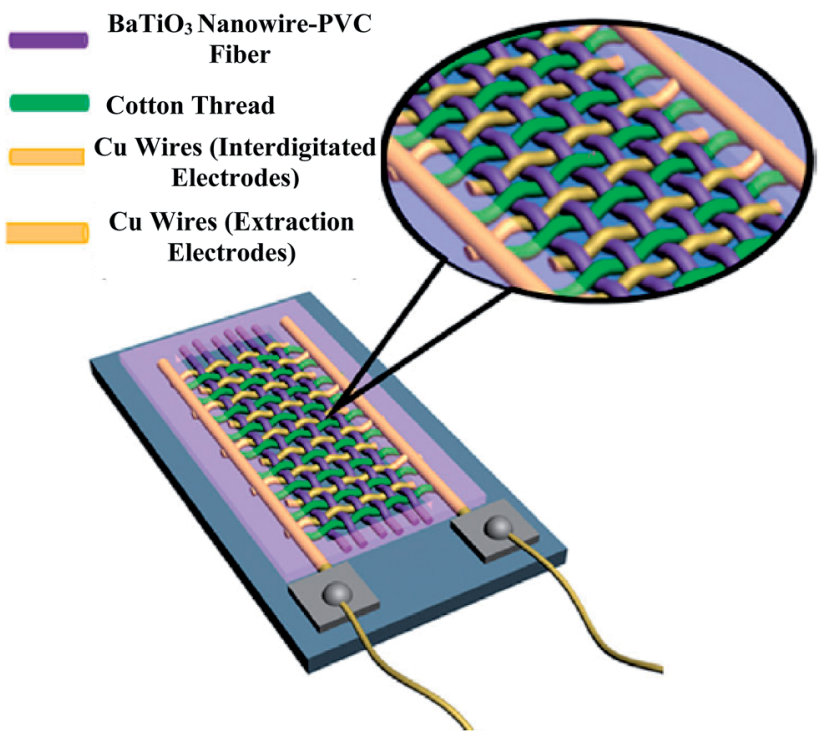

(b)

Fig. 19 (a) Photograph of a fabric nanogenerator (FNG). (b) Structure of the FNG. ${ }^{248}$

\section{Smart wearable systems}

Smart clothes can be woven by integrating NSs with functional fibers. They are used to power sensors with low power consumption by harvesting mechanical energy from the motions of the human body. ${ }^{6,246,268,269}$ A photograph of a fabric generator together with its structure is shown in Fig. 19.

A self-powered strain sensor could be developed by using smart cloth composed of an FBHNG. A self-powered alert system could also be designed by using the rectified output of an FBHNG. ${ }^{242}$

\section{Transparent and flexible devices}

Optical devices such as light-emitting diodes and photovoltaics make use of thin metal or metal oxide films as transparent conductors. $^{270}$ These transparent metal-based films possess a limited range of applications because of their reduced elasticity, chemical instability and high cost. ${ }^{271,272}$ CNTs have replaced metal-based flexible thin films owing to their effective optical, mechanical and electrical properties and have been utilized in several flexible optical devices. ${ }^{273-275}$ The performance of CNTs has been limited by their diameter-dependent electrical properties, difficulties in the separation of metallic and semiconducting CNTs and unevenness, which have limited their application in flexible devices.

Yang et al. (2009) designed an SWG-based flexible piezoelectric $\mathrm{ZnO}$ nanowire, which converted biomechanical energy into a suitable electrical response. ${ }^{8}$ It had the ability to convert
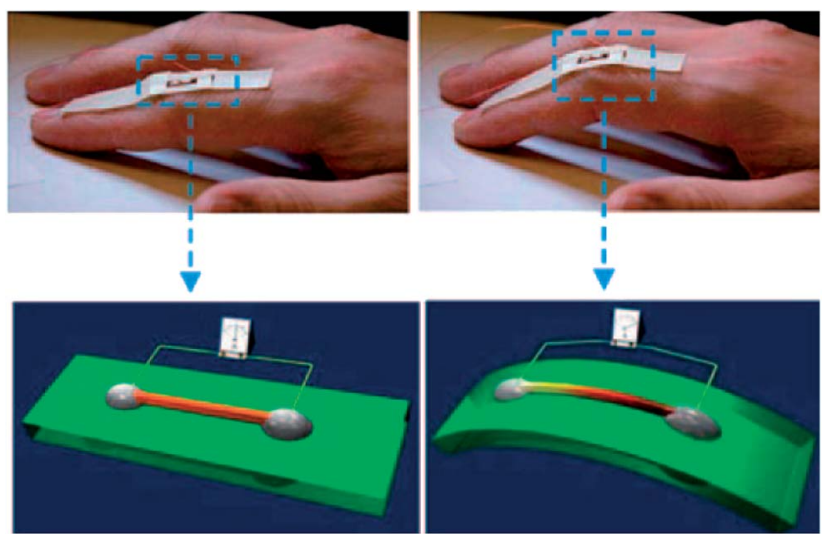

(a)
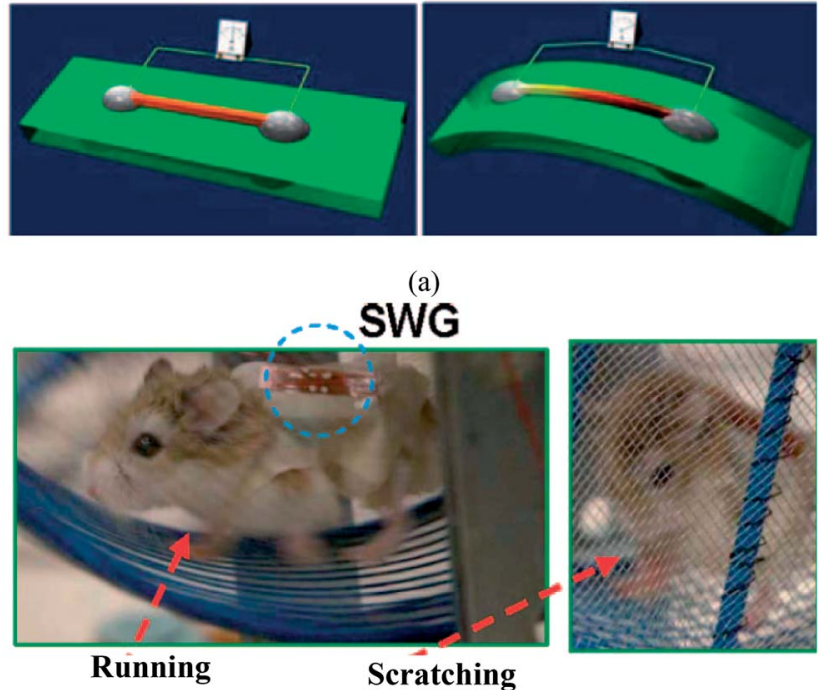

(b)

Fig. 20 Energy harvesting using an SWG from (a) an oscillating human index finger and (b) a live hamster. ${ }^{8}$ 


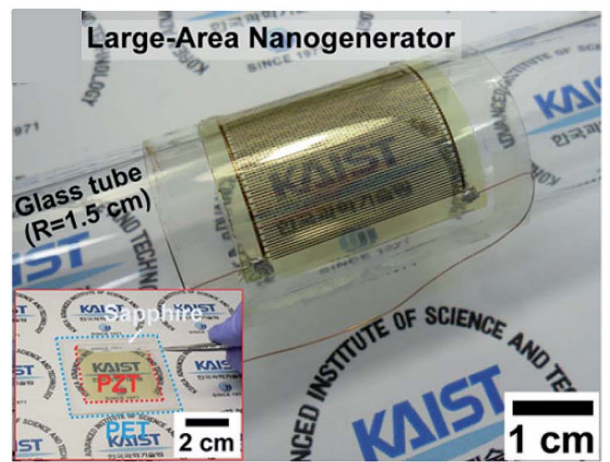

Fig. 21 Fabricated large-area thin PZT film NG on a curved glass tube. The inset shows a thin PZT film on a PET substrate formed by an LLO process. ${ }^{276}$

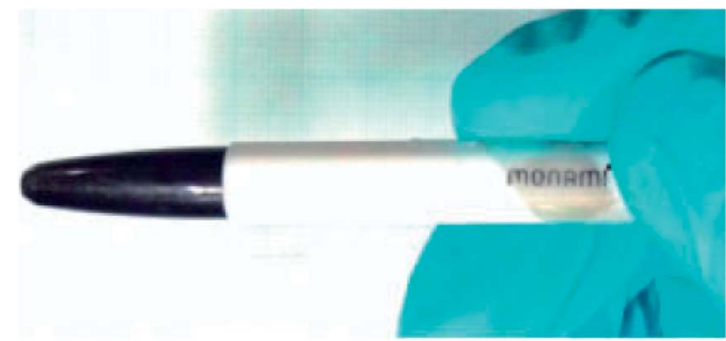

Fig. 22 Fully rollable graphene-based NG. ${ }^{103}$

the tapping of a human finger and the motion of a running live hamster (Campbell's dwarf) into electricity. An output voltage of up to $\sim 0.1-0.15 \mathrm{~V}$ could be generated by integrating four SWGs in series. The harvesting of energy from the NG attached to a human finger or running hamster is depicted in Fig. 20.

Kwi et al. (2014) fabricated a highly efficient, flexible and lightweight thin PZT film NG by a conventional sol-gel method. ${ }^{276}$ An LLO process was used to transfer the thin film from a sapphire substrate onto a plastic substrate without mechanical damage (Fig. 21). The thin film NG could generate an output voltage and current density of $\sim 200 \mathrm{~V}$ and $150 \mu \mathrm{A}$ $\mathrm{cm}^{-2}$, respectively, upon bending the film. The power generated by the NG from human finger motions was used to drive 100 blue LEDs with no interfacing circuitry.

The transparency of the device can be increased by substituting the indium tin oxide (ITO) electrode with a graphene layer without affecting the cost. ${ }^{103}$ The graphene-based NG is shown in Fig. 22. Graphene displayed improved performance owing to its better electrical and mechanical properties, ${ }^{277-280}$ such as high mobility ${ }^{277}$ at room temperature, high optical transmittance, improved chemical stability and relatively high mechanical elasticity, ${ }^{280}$ which shows that it is a favorable option for applications in a wide range of fields such as nanoelectronics, optoelectronics and spintronics. ${ }^{281-284}$ The major drawback faced by graphene is its time-consuming fabrication process, which may lead to graphene sheets with low optical and electrical quality because of structural defects and low interlayer junction contact resistance.

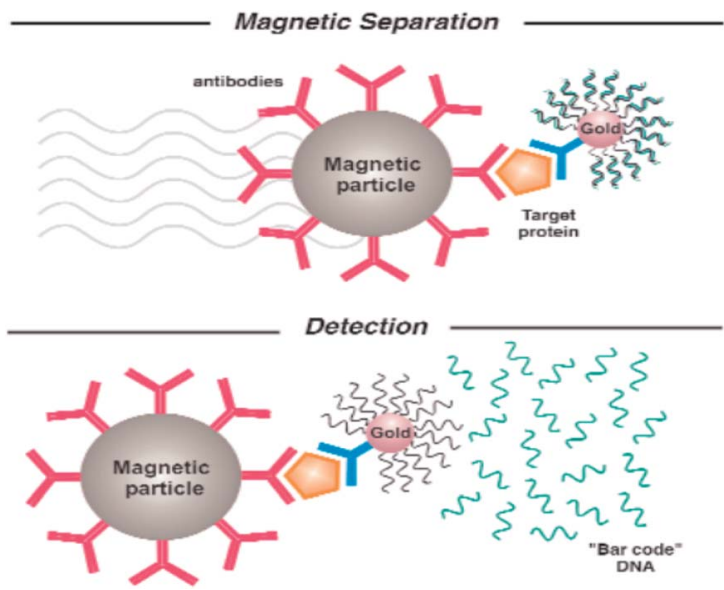

Fig. 23 Detection of protein by means of nanoparticles. ${ }^{294}$

PENGs with exceptionally high flexibility can be used as energy-scavenging devices in various applications, which include wireless sensors and self-powered NEMS-based devices. ${ }^{285-287}$

\section{E. Biomedical applications}

Nanostructures such as nanopatterns, nanochannels, nanotubes and nanoparticles are utilized for a range of biomedical applications. Fluorescent biological labeling and tagging, ${ }^{288-290}$

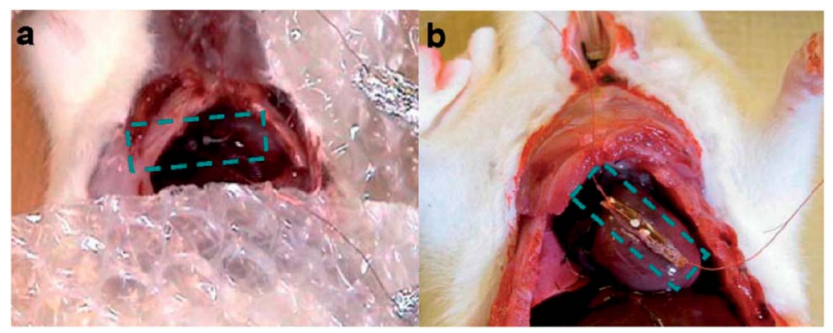

Fig. 24 Energy harvesting from the breath and heartbeat of a live rat using an SWG attached to (a) the rat's diaphragm and (b) the rat's heart. $^{7}$

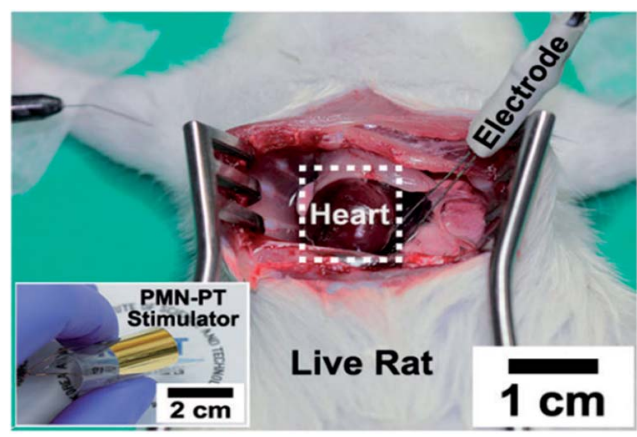

Fig. 25 Photograph of a medical experiment with an NG attached to the heart of a living rat. The inset shows a snapshot of flexible PMN-PT thin film stimulator ${ }^{302}$ 
drug and gene delivery, ${ }^{291,292}$ and the biodetection of pathogens $^{293}$ and proteins ${ }^{294}$ have been performed by means of nanoparticles. The detection of protein molecules with the help of nanoparticles is illustrated in Fig. 23.

Nanochannels and nanotubes have been employed in various areas such as structural studies of $\mathrm{DNA}^{74}$ and tissue engineering. ${ }^{295,296}$ Magnetic nanoparticles play a wider range of roles in medical fields such as the separation and purification of biological molecules and cells, ${ }^{297}$ contrast enhancement in magnetic resonance imaging (MRI) ${ }^{298}$ and

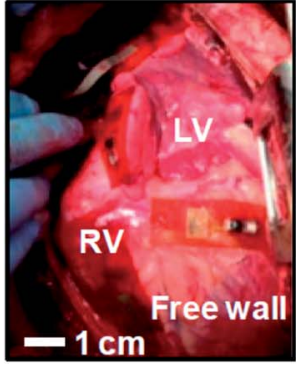

(a)

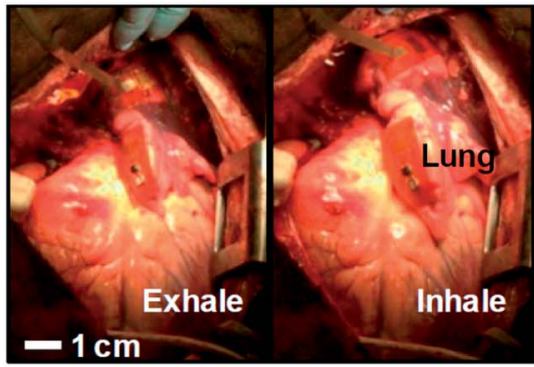

(d)

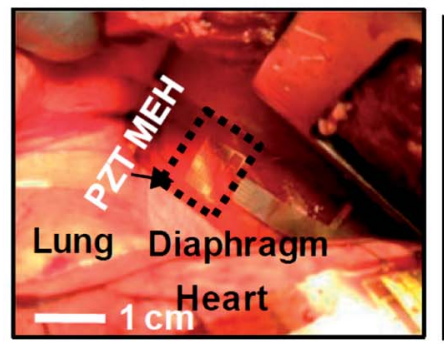

(g)

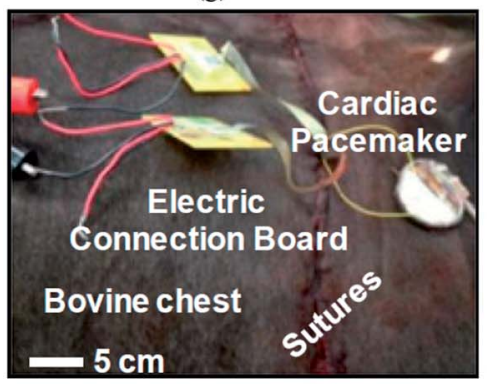

(i)

Fig. 26 Photographs of PZT MEHs on the (a) RV, LV, and free wall of a bovine heart, (b) bovine heart during expansion, (c) bovine heart during relaxation, (d) bovine lung during exhalation, (e) bovine lung during inhalation, (f) at different angles of orientation, (g) bovine diaphragm, (h) open chest and (i) closed chest. ${ }^{303}$
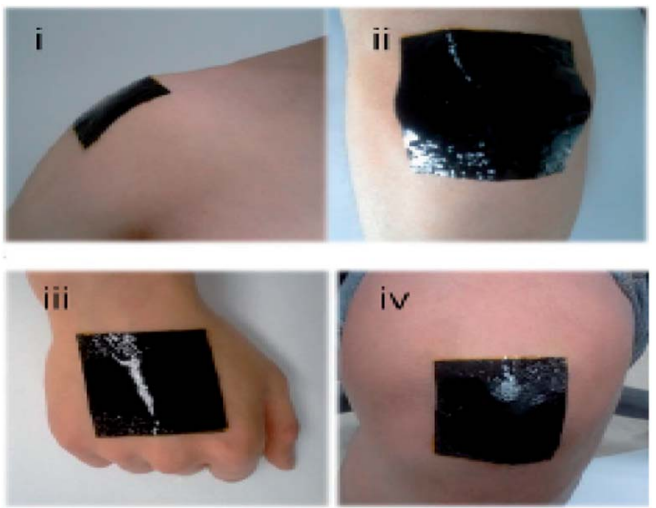

Fig. 27 Photographic images of the HSNG at various locations on the human body (i) shoulder (ii) elbow (iii) dorsal side of hand (iv) knee showing the good compatibility of the device. ${ }^{251}$

hyperthermia (destruction of tumor cells by means of heating), ${ }^{299}$ and many phagokinetic studies ${ }^{\mathbf{3 0 0}}$ are currently under investigation.

\section{F. Implantable devices}

The toxicity of chemical batteries fails to meet the power supply needs of implantable micro/nanoscale devices, which are widely used in biomedical applications. Yu et al. (2009) suggested the use of $\mathrm{ZnO}$ nanowires for in vivo applications because they are biologically safe and biodegradable..$^{301}$

Zhou et al. (2010) reported an SWG-based in vivo nanogenerator that can generate electrical energy when attached to the diaphragm and heart of a live rat. ${ }^{7}$ This in vivo generator is capable of capturing vibrations from the periodic expansions and contractions of the diaphragm (breath) and heartbeat of a living rat and generates suitable electrical output, as shown in Fig. 24. These non-toxic nanogenerators have become an essential step in the evolution of implantable self-powered nanodevices.

Geon et al. (2014) developed a flexible efficient singlecrystalline thin PMN-PT film on a plastic substrate, which was used to design a self-powered cardiac pacemaker. ${ }^{302}$ This harvester generated an output voltage of $8.2 \mathrm{~V}$ and a current of $145 \mu \mathrm{A}$ when it was subjected to periodic mechanical motions of bending and unbending. The experimental investigation of the fabricated PMN-PT-based NG is illustrated in Fig. 25.

Canan et al. (2014) proposed an implantable NG that could act as a power source for continuous health-monitoring devices, as well as non-biomedical devices, such as heart rate monitors, pacemakers, cardioverter-defibrillators and neural stimulators. ${ }^{303}$ The operation of the device using the natural contractile and relaxation motions of the heart, lung and diaphragm was demonstrated on animal models (Fig. 26) of which the organs are comparable in size to human organs.

\section{G. Security systems}

PENGs hold notable potential importance as energy-harvesting power sources and have been used as several types of motion 
sensor for transport monitoring, ${ }^{304}$ biomedical sensors, ${ }^{305}$ wireless sensors ${ }^{306}$ and tactile sensors. ${ }^{250}$

The hybrid energy-scavenging NG reported by Keun et al. (2014) displayed high compatibility with different parts of the human body and hence represents a better choice for wearable devices, robotics and biomedical applications with exceptional sustainability. ${ }^{251}$ The integrability of the hybrid device is depicted in Fig. 27. Hence, PENG-based energy scavengers pave the way for the development of self-powered nurse call systems and panic systems for providing continuous security. They are very helpful for the development of security systems for the sake of people at risk.

Fire alarm and detection systems (FADs), closed-circuit television systems (CCTV), burglar alarm systems (SOT), security systems and their integration can also be made possible by developing a self-powered system with the help of this piezoelectric nanoscale energy-scavenging device. The consumption of power can be reduced by a huge amount via the integration of these PENGs into our day-to-day appliances.

\section{Conclusions}

This paper has focused on a comprehensive survey of piezoelectric nanogenerators. The operating modes of harvesters based on the direction of the applied force have been discussed. Among the various available configurations, VINGs are predominantly in demand because of their outstanding properties such as enhanced output performance, low cost and easy fabrication techniques, whereas LINGs need costly fabrication techniques and NEGs require complex as well as expensive synthesis methodologies. Reductions in the quality and stability of devices due to scaling can be rectified by using efficient fabrication techniques. The bottom-up approach is superior to the top-down approach owing to its reduced processing time, low cost and synthesis of high-quality nanostructures. The use of the inorganic materials used for the design of early PENGs has been limited because of their low formability and low structural stability. Polymers have been used for the design of lightweight NGs. Various research studies have been performed for the development of piezoelectric materials with improved piezoelectric performance, as well as lead-free piezoelectric materials. Despite the increased piezoelectric constant of leadbased materials, their consumption has diminished owing to their toxicity. $\mathrm{ZnO}$ acts as a universal material with coupled semiconducting and piezoelectric properties owing to its unsurpassed attributes such as biocompatibility, chemical stability, ample availability, and affordability. Zinc stannate can replace $\mathrm{ZnO}$ owing to its extremely high piezoelectric coefficient and attractive optical properties with high transparency. Titanium dioxide is a good material of choice in the field of energy harvesting because of its easy synthesis procedure, chemical stability and high energy conversion efficiency. The energy conversion efficiency of PENGs can be improved by various strategies such as doping, temperature control, suppression of screening effects, diameter reduction, host layer addition, topological optimization, surface passivation, etc. Hybrid energy harvesters can be developed either by integrating different energy-harvesting mechanisms or by combining different piezoelectric materials, which results in the long-term continuous availability of power. Noteworthy applications of PENGs in various fields have been reported. PENGs have led to the development of many self-powered sensors, consumer electronic devices, wearable sensors and ubiquitous security systems, which act as stepping stones to a green environment.

\section{References}

1 S. Newman, The final energy crisis, Pluto, 2008.

2 G. Zhu, Z. H. Lin, Q. Jing, P. Bai, C. Pan, Y. Yang, Y. Zhou and Z. L. Wang, Toward large-scale energy harvesting by a nanoparticle-enhanced triboelectric nanogenerator, Nano Lett., 2013, 13(2), 847-853.

3 M. P. Lu, J. Song, M. Y. Lu, M. T. Chen, Y. Gao, L. J. Chen and Z. L. Wang, Piezoelectric nanogenerator using p-type ZnO nanowire arrays, Nano Lett., 2009, 9(3), 1223-1227.

4 F. Gao, W. Li, X. Wang, X. Fang and M. Ma, A self-sustaining pyroelectric nanogenerator driven by water vapor, Nano Energy, 2016, 22, 19-26.

5 C. Hu, L. Cheng, Z. Wang, Y. Zheng, S. Bai and Y. Qin, A transparent antipeep piezoelectric nanogenerator to harvest tapping energy on screen, Small, 2016, 12(10), 1315-1321.

6 Z. Li and Z. L. Wang, Air/Liquid-Pressure and HeartbeatDriven Flexible Fiber Nanogenerators as a Micro/NanoPower Source or Diagnostic Sensor, Adv. Mater., 2011, 23(1), 84-89.

7 Z. Li, G. Zhu, R. Yang, A. C. Wang and Z. L. Wang, Muscledriven in vivo nanogenerator, Adv. Mater., 2010, 22(23), 2534-2537.

8 R. Yang, Y. Qin, C. Li, G. Zhu and Z. L. Wang, Converting biomechanical energy into electricity by a musclemovement-driven nanogenerator, Nano Lett., 2009, 9(3), 1201-1205.

9 Z. L. Wang and J. Song, Piezoelectric nanogenerators based on zinc oxide nanowire arrays, Science, 2006, 312(5771), 242-246.

10 D. Grützmacher, M. Mikulics and H. Hardtdegen, Lowenergy consumption nano-opto-electronics based on IIInitride-LED mesoscopic structures, in Solid-State Device Research Conference (ESSDERC), 2016 46th European, IEEE, 2016, pp. 327-329.

11 W. Tian, W. Li, X. Liu and Y. Wang, Molecular Dynamics Study on the Resonance Properties of a Nano Resonator Based on a Graphene Sheet with Two Types of Vacancy Defects, Appl. Sci., 2017, 7(1), 79.

12 S. Chinnakkannu Vijayakumar, K. Venkatakrishnan and B. Tan, SERS Active Nanobiosensor Functionalized by Self-Assembled 3D Nickel Nanonetworks for Glutathione Detection, ACS Appl. Mater. Interfaces, 2017, 9(6), 50775091.

13 L. Gu, N. Cui, L. Cheng, Q. Xu, S. Bai, M. Yuan, W. Wu, J. Liu, Y. Zhao, F. Ma and Y. Qin, Flexible fiber nanogenerator with $209 \mathrm{~V}$ output voltage directly powers a light-emitting diode, Nano Lett., 2012, 13(1), 91-94. 
14 J. Kwon, W. Seung, B. K. Sharma, S. W. Kim and J. H. Ahn, A high performance PZT ribbon-based nanogenerator using graphene transparent electrodes, Energy Environ. Sci., 2012, 5(10), 8970-8975.

15 J. H. Jung, M. Lee, J. I. Hong, Y. Ding, C. Y. Chen, L. J. Chou and Z. L. Wang, Lead-free $\mathrm{NaNbO}_{3}$ nanowires for a high output piezoelectric nanogenerator, ACS Nano, 2011, 5(12), 10041-10046.

16 C. Falconi, G. Mantini, A. D'Amico and V. Ferrari, Modeling of piezoelectric nanodevices, in Piezoelectric Nanomaterials for Biomedical Applications, Springer, Berlin, Heidelberg, 2012, pp. 93-133.

17 G. Mantini, Y. Gao, A. D'Amico, C. Falconi and Z. L. Wang, Equilibrium piezoelectric potential distribution in a deformed ZnO nanowire, Nano Res., 2009, 2(8), 624-629.

18 B. J. Hansen, Y. Liu, R. Yang and Z. L. Wang, Hybrid nanogenerator for concurrently harvesting biomechanical and biochemical energy, ACS Nano, 2010, 4(7), 3647-3652.

19 Y. Yang, H. Zhang, Z. H. Lin, Y. Liu, J. Chen, Z. Lin, Y. S. Zhou, C. P. Wong and Z. L. Wang, A hybrid energy cell for self-powered water splitting, Energy Environ. Sci., 2013, 6(8), 2429-2434.

20 S. Xu, Y. Qin, C. Xu, Y. Wei, R. Yang and Z. L. Wang, Selfpowered nanowire devices, Nat. Nanotechnol., 2010, 5(5), 366-373.

21 S. Hasegawa, S. Nishida, T. Yamashita and H. Asahi, Field electron emission from polycrystalline GaN nanorods, Journal of Ceramic Processing Research, 2005, 6(3), 245-249.

22 C. A. Mead, Surface barriers on ZnSe and ZnO, Phys. Lett., 1965, 18(3), 218.

23 C. Xu, P. Shin, L. Cao and D. Gao, Preferential growth of long $\mathrm{ZnO}$ nanowire array and its application in dyesensitized solar cells, J. Phys. Chem. C, 2009, 114(1), 125129.

24 W. Lu and J. Xiang, Semiconductor Nanowires: From NextGeneration Electronics to Sustainable Energy, Royal Society of Chemistry, 2015, ISBN: 978-1-84973-815.

25 K. Momeni, G. M. Odegard and R. S. Yassar, Nanocomposite electrical generator based on piezoelectric zinc oxide nanowires, J. Appl. Phys., 2010, 108(11), 114303.

26 K. Momeni and S. M. Mortazavi, Optimal aspect ratio of zinc oxide nanowires for a nanocomposite electrical generator, J. Comput. Theor. Nanosci., 2012, 9(10), 16701674.

27 X. Wang, J. Song, J. Liu and Z. L. Wang, Direct-current nanogenerator driven by ultrasonic waves, Science, 2007, 316(5821), 102-105.

28 M. Y. Choi, D. Choi, M. J. Jin, I. Kim, S. H. Kim, J. Y. Choi, S. Y. Lee, J. M. Kim and S. W. Kim, Mechanically powered transparent flexible charge-generating nanodevices with piezoelectric ZnO nanorods, Adv. Mater., 2009, 21(21), 2185-2189.

29 D. Choi, M. Y. Choi, H. J. Shin, S. M. Yoon, J. S. Seo, J. Y. Choi, S. Y. Lee, J. M. Kim and S. W. Kim, Nanoscale networked single-walled carbon-nanotube electrodes for transparent flexible nanogenerators, J. Phys. Chem. C, 2009, 114(2), 1379-1384.

30 X. Wang, J. Song, J. Liu and Z. L. Wang, Direct-current nanogenerator driven by ultrasonic waves, Science, 2007, 316(5821), 102-105.

31 M. S. Al-Ruqeishi, T. Mohiuddin, B. Al-Habsi, F. AlRuqeishi, A. Al-Fahdi and A. Al-Khusaibi, Piezoelectric nanogenerator based on ZnO nanorods, Arabian J. Chem., 2016, DOI: 10.1016/j.arabjc.2016.12.010.

32 D. Choi, M. Y. Choi, H. J. Shin, S. M. Yoon, J. S. Seo, J. Y. Choi, S. Y. Lee, J. M. Kim and S. W. Kim, Nanoscale networked single-walled carbon-nanotube electrodes for transparent flexible nanogenerators, J. Phys. Chem. C, 2009, 114(2), 1379-1384.

33 P. Deb, H. Kim, V. Rawat, M. Oliver, S. Kim, M. Marshall, E. Stach and T. Sands, Faceted and vertically aligned GaN nanorod arrays fabricated without catalysts or lithography, Nano Lett., 2005, 5(9), 1847-1851.

34 Y. Yang, Y. Xia, W. Huang, J. Zheng and Z. Li, Fabrication of nano-network gold films via anodization of gold electrode and their application in SERS, J. Solid State Electrochem., 2012, 16(4), 1733-1739.

35 K. Kim, Y. M. Choi, D. G. Gweon and M. G. Lee, A novel laser micro/nano-machining system for FPD process, J. Mater. Process. Technol., 2008, 201(1), 497-501.

36 A. De Luca, F. Ritton, M. Gelly, J. Buckley and M. Heitzman, Silicon dry etching process for high topography sub-micro and nano-devices, ECS Trans., 2008, 16(14), 45-50.

37 B. Gorzolnik, P. Mela and M. Moeller, Nano-structured micropatterns by combination of block copolymer selfassembly and UV photolithography, Nanotechnology, 2006, 17(19), 5027.

38 U. D. Zeitner, T. Harzendorf, F. Fuchs, M. Banasch, H. Schmidt and E. B. Kley, Efficient fabrication of complex nano-optical structures by E-beam lithography based on character projection, in SPIE MOEMS-MEMS, International Society for Optics and Photonics, 2014, p. $89740 \mathrm{G}$.

39 N. Das, A. Karar, M. Vasiliev, C. L. Tan, K. Alameh and Y. T. Lee, Analysis of nano-grating-assisted light absorption enhancement in metal-semiconductor-metal photodetectors patterned using focused ion-beam lithography, Opt. Commun., 2011, 284(6), 1694-1700.

40 E. Di Fabrizio, R. Fillipo, S. Cabrini, R. Kumar, F. Perennes, M. Altissimo, L. Businaro, D. Cojac, L. Vaccari, M. Prasciolu and P. Candeloro, X-ray lithography for micro-and nanofabrication at ELETTRA for interdisciplinary applications, J. Phys.: Condens. Matter, 2004, 16(33), S3517.

41 R. D. Piner, J. Zhu, F. Xu, S. Hong and C. A. Mirkin, Dip-pen nanolithography, Science, 1999, 283(5402), 661-663.

42 I. Brodie and J. J. Muray, Particle beams: sources, optics, and interactions, in The Physics of Microfabrication, Springer, US, 1982, pp. 79-225.

43 L. F. Alwitt, Suspense and advertising responses, J. Consum. Psychol., 2002, 12(1), 35-49. 
44 C. Cardinaud, M. C. Peignon and P. Y. Tessier, Plasma etching: principles, mechanisms, application to microand nano-technologies, Appl. Surf. Sci., 2000, 164(1), 72-83.

45 K. Mitsuishi, M. Shimojo, M. Tanaka and K. Furuya, Nanofabrication using electron-beam-induced deposition combined with low energy ion milling, Nucl. Instrum. Methods Phys. Res., Sect. B, 2006, 242(1), 244-246.

46 G. Sun, T. Gao, X. Zhao and H. Zhang, Fabrication of micro/ nano dual-scale structures by improved deep reactive ion etching, J. Micromech. Microeng., 2010, 20(7), 075028.

47 H. Bahadur, A. K. Srivastava, D. Haranath, H. Chander, A. Basu, S. B. Samanta, K. N. Sood, R. Kishore, R. K. Sharma, V. Bhatt and P. Pal, Nano-structured ZnO films by sol-gel process, Indian J. Pure Appl. Phys., 2007, 45, 395-399.

48 J. T. Mckinnon, A. M. Herring and B. D. Mccloskey, Laser pyrolysis method for producing carbon nano-spheres, US Pat., 7,601,321, 2009.

49 S. Manna, J. W. Kim, Y. Takahashi, O. G. Shpyrko and E. E. Fullerton, Synthesis of single-crystalline anisotropic gold nano-crystals via chemical vapor deposition, J. Appl. Phys., 2016, 119(17), 174301.

50 C. Zhang, J. Li, C. Shi, E. Liu, X. Du, W. Feng and N. Zhao, The efficient synthesis of carbon nano-onions using chemical vapor deposition on an unsupported Ni-Fe alloy catalyst, Carbon, 2011, 49(4), 1151-1158.

51 L. Jia and F. Gitzhofer, Induction plasma synthesis of nanostructured SOFCs electrolyte using solution and suspension plasma spraying: a comparative study, J. Therm. Spray Technol., 2010, 19(3), 566-574.

$52 \mathrm{~J}$. Chung, J. Lee and S. Lim, Annealing effects of $\mathrm{ZnO}$ nanorods on dye-sensitized solar cell efficiency, Phys. B, 2010, 405(11), 2593-2598.

53 X. Fang, Y. Li, S. Zhang, L. Bai, N. Yuan and J. Ding, The dye adsorption optimization of $\mathrm{ZnO}$ nanorod-based dyesensitized solar cells, Sol. Energ., 2014, 105, 14-19.

54 Z. Qin, G. Zhang, Q. Liao, Y. Qiu, Y. Huang and Y. Zhang, Influences of low temperature thermal treatment on $\mathrm{ZnO}$ nanowire arrays and nanoparticles based flexible dyesensitized solar cells, Colloids Surf., A, 2012, 402, 127131.

55 F. D. Nayeri, E. A. Soleimani and F. Salehi, Synthesis and characterization of $\mathrm{ZnO}$ nanowires grown on different seed layers: the application for dye-sensitized solar cells, Renewable Energy, 2013, 60, 246-255.

56 M. T. Htay, Y. Hashimoto and K. Ito, Growth of $\mathrm{ZnO}$ submicron single-crystalline platelets, wires, and rods by ultrasonic spray pyrolysis, Jpn. J. Appl. Phys., Part 1, 2007, 46(1), 440.

57 M. M. Yusoff, M. H. Mamat, M. F. Malek, A. B. Suriani, A. Mohamed, M. K. Ahmad, S. A. Alrokayan, H. A. Khan and M. Rusop, Growth of titanium dioxide nanorod arrays through the aqueous chemical route under a novel and facile low-cost method, Mater. Lett., 2016, 164, 294-298.

58 G. Kalita, K. Wakita and M. Umeno, Low temperature growth of graphene film by microwave assisted surface wave plasma CVD for transparent electrode application, RSC Adv., 2012, 2(7), 2815-2820.

59 S. Xu, C. Cheng, W. Guo, Y. He, R. Huang, S. Du and $\mathrm{N}$. Wang, Tuning the optical and electrical properties of hydrothermally grown $\mathrm{ZnO}$ nanowires by sealed post annealing treatment, RSC Adv., 2013, 160, 41-46.

60 Z. Kang, Y. Gu, X. Yan, Z. Bai, Y. Liu, S. Liu, X. Zhang, Z. Zhang, X. Zhang and Y. Zhang, Enhanced photoelectrochemical property of ZnO nanorods array synthesized on reduced graphene oxide for self-powered biosensing application, Biosens. Bioelectron., 2015, 64, 499-504.

61 W. A. Satoshi, T. Suzuki and N. O. Tatsuo, Preparation of barium titanate fine particles by hydrothermal method and their characterization, J. Ceram. Soc. Jpn., 1995, 103(1204), 1220-1227.

$62 \mathrm{H}$. Lu, X. Zhai, W. Liu, M. Zhang and M. Guo, Electrodeposition of hierarchical ZnO nanorod arrays on flexible stainless steel mesh for dye-sensitized solar cell, Thin Solid Films, 2015, 586, 46-53.

63 Y. Chae, J. T. Park, J. K. Koh, J. H. Kim and E. Kim, All-solid, flexible solar textiles based on dye-sensitized solar cells with ZnO nanorod arrays on stainless steel wires, Mater. Sci. Eng., B, 2013, 178(17), 1117-1123.

64 S. Zhu, L. Shan, X. Tian, X. Zheng, D. Sun, X. Liu, L. Wang and Z. Zhou, Hydrothermal synthesis of oriented $\mathrm{ZnO}$ nanorod-nanosheets hierarchical architecture on zinc foil as flexible photoanodes for dye-sensitized solar cells, Ceram. Int., 2014, 40(8), 11663-11670.

65 J. Du, F. Bittner, D. S. Hecht, C. Ladous, J. Ellinger, T. Oekermann and M. Wark, A carbon nanotube-based transparent conductive substrate for flexible $\mathrm{ZnO}$ dyesensitized solar cells, Thin Solid Films, 2013, 531, 391-397.

66 H. W. Chen, C. Y. Lin, Y. H. Lai, J. G. Chen, C. C. Wang, C. W. Hu, C. Y. Hsu, R. Vittal and K. C. Ho, Electrophoretic deposition of $\mathrm{ZnO}$ film and its compression for a plastic based flexible dye-sensitized solar cell, J. Power Sources, 2011, 196(10), 4859-4864.

$67 \mathrm{H}$. T. Chou and H. C. Hsu, The effect of annealing temperatures to prepare $\mathrm{ZnO}$ seeds layer on $\mathrm{ZnO}$ nanorods array/TiO 2 nanoparticles photoanode, SolidState Electron., 2016, 116, 15-21.

68 R. Musembi, B. Aduda, J. Mwabora, M. Rusu, K. Fostiropoulos and M. Lux-Steiner, Effect of Recombination on Series Resistance in eta Solar Cell Modified with $\operatorname{In}(\mathrm{OH})_{x} \mathrm{~S}_{y}$ Buffer Layer, Int. J. Energ. Eng., 2013, 3(3), 183-189.

69 M. Zafar, M. Zafar, M. Naeem Awais, M. Naeem Awais, M. Asif, M. Asif, A. Razaq, A. Razaq, G. Amin and G. Amin, Fabrication and characterization of piezoelectric nanogenerator based on $\mathrm{Al} / \mathrm{ZnO} / \mathrm{Au}$ structure, Microelectron. Int., 2017, 34(1), 35-39.

70 M. Ahmad, M. A. Iqbal, J. Kiely, R. Luxton and M. Jabeen, Enhanced output voltage generation via $\mathrm{ZnO}$ Nanowires $(50 \mathrm{~nm})$ : Effect of diameter thinning on voltage enhancement, J. Phys. Chem. Solids, 2017, 281-285. 
71 R. L. Twite and G. P. Bierwagen, Review of alternatives to chromate for corrosion protection of aluminum aerospace alloys, Prog. Org. Coat., 1998, 33(2), 91-100.

72 M. Lee, C. Y. Chen, S. Wang, S. N. Cha, Y. J. Park, J. M. Kim, L. J. Chou and Z. L. Wang, A hybrid piezoelectric structure for wearable nanogenerators, Adv. Mater., 2012, 24(13), 1759-1764.

73 M. J. Madou, Fundamentals of microfabrication: the science of miniaturization, CRC press, 2002.

74 R. Mahtab, J. P. Rogers and C. J. Murphy, Protein-sized quantum dot luminescence can distinguish between "straight", "bent", and "kinked" oligonucleotides, J. Am. Chem. Soc., 1995, 117(35), 9099-9100.

75 S. G. Ullattil and P. Periyat, Sol-Gel Synthesis of Titanium Dioxide, in Sol-Gel Materials for Energy, Environment and Electronic Applications, Springer International Publishing, 2017, pp. 271-283.

76 A. Khan, A. Rashid, R. Younas and R. Chong, A chemical reduction approach to the synthesis of copper nanoparticles, Int. Nano Lett., 2016, 6(1), 21-26.

77 N. Herlin-Boime, J. Vicens, C. Dufour, F. Ténégal, C. Reynaud and R. Rizk, Flame temperature effect on the structure of SiC nanoparticles grown by laser pyrolysis, $J$. Nanopart. Res., 2004, 6(1), 63-70.

78 C. D. Scott, S. Arepalli, P. Nikolaev and R. E. Smalley, Growth mechanisms for single-wall carbon nanotubes in a laser-ablation process, Appl. Phys. A: Mater. Sci. Process., 2001, 72(5), 573-580.

79 D. P. Yu, C. S. Lee, I. Bello, X. S. Sun, Y. H. Tang, G. W. Zhou, Z. G. Bai, Z. Zhang and S. Q. Feng, Synthesis of nano-scale silicon wires by excimer laser ablation at high temperature, Solid State Commun., 1998, 105(6), 403-407.

80 C. Dhand, N. Dwivedi, X. J. Loh, A. N. Ying, N. K. Verma, R. W. Beuerman, R. Lakshminarayanan and S. Ramakrishna, Methods and strategies for the synthesis of diverse nanoparticles and their applications: a comprehensive overview, RSC Adv., 2015, 5(127), 105003-105037.

81 K. S. Cho, D. H. Kim, Y. H. Kim, J. Nah and H. K. Kim, Lidoped $\mathrm{Cu}_{2} \mathrm{O} / \mathrm{ZnO}$ heterojunction for flexible and semitransparent piezoelectric nanogenerators, Ceram. Int., 2017, 43(2), 2279-2287.

82 X. Chen, Q. Xu, S. Bai and Y. Qin, A self-powered sensor with super-hydrophobic nanostructure surfaces for synchronous detection and electricity generation, Nano Energy, 2017, 33, 288-292.

83 F. Kayaci, C. Ozgit Akgun, I. Donmez, N. Biyikli and T. Uyar, Polymer-inorganic core-shell nanofibers by electrospinning and atomic layer deposition: flexible nylon-ZnO core-shell nanofiber mats and their photocatalytic activity, ACS Appl. Mater. Interfaces, 2012, 4(11), 6185-6194.

84 A. Majeed, J. He, L. Jiao, X. Zhong and Z. Sheng, Surface properties and biocompatibility of nanostructured $\mathrm{TiO}_{2}$ film deposited by RF magnetron sputtering, Nanoscale Res. Lett., 2015, 10, 56, DOI: 10.1186/s11671-015-0732-7.
85 Z. H. Lin, Z. Yang and H. T. Chang, Preparation of fluorescent tellurium nanowires at room temperature, Cryst. Growth Des., 2007, 8(1), 351-357.

86 M. M. Abolhasani, K. Shirvanimoghaddam and M. Naebe, PVDF/graphene composite nanofibers with enhanced piezoelectric performance for development of robust nanogenerators, Compos. Sci. Technol., 2017, 138, 49-56.

87 H. H. Fan, C. C. Jin, Y. Wang, H. L. Hwang and Y. F. Zhang, Structural of BCTZ nanowires and high performance BCTZbased nanogenerator for biomechanical energy harvesting, Ceram. Int., 2017, 43(8), 5875-5880.

88 C. Ribeiro, V. Sencadas, J. L. Ribelles and S. LancerosMéndez, Influence of processing conditions on polymorphism and nanofiber morphology of electroactive poly(vinylidene fluoride) electrospun membranes, Soft Mater., 2010, 8(3), 274-287.

89 J. Nunes-Pereira, V. Sencadas, V. Correia, J. G. Rocha and S. Lanceros-Méndez, Energy harvesting performance of piezoelectric electrospun polymer fibers and polymer/ ceramic composites, Sens. Actuators, A, 2013, 196, 55-62.

90 A. Greiner and J. H. Wendorff, Electrospinning: a fascinating method for the preparation of ultrathin fibers, Angew. Chem., Int. Ed., 2007, 46(30), 5670-5703.

91 W. E. Teo and S. Ramakrishna, A review on electrospinning design and nanofibre assemblies, Nanotechnology, 2006, 17(14), R89.

92 Y. Lu, R. Ganguli, C. A. Drewien, M. T. Anderson, C. J. Brinker, W. Gong, Y. Guo, H. Soyez, B. Dunn, M. H. Huang and J. I. Zink, Continuous formation of supported cubic and hexagonal mesoporous films by solgel dip-coating, Nature, 1997, 389(6649), 364-368.

93 I. Dakua and N. Afzulpurkar, Piezoelectric energy generation and harvesting at the nano-scale: materials and devices, Nanomater. Nanotechnol., 2013, 3, 21.

94 Z. Wang, X. Pan, Y. He, Y. Hu, H. Gu and Y. Wang, Piezoelectric nanowires in energy harvesting applications, Adv. Mater. Sci. Eng., 2015, 165631.

$95 \mathrm{M}$. Minary-Jolandan and M. F. Yu, Nanoscale characterization of isolated individual type I collagen fibrils: polarization and piezoelectricity, Nanotechnology, 2009, 20(8), 085706.

96 M. Minary-Jolandan and M. F. Yu, Uncovering nanoscale electromechanical heterogeneity in the subfibrillar structure of collagen fibrils responsible for the piezoelectricity of bone, ACS Nano, 2009, 3(7), 1859-1863.

97 D. Denning, M. V. Paukshto, S. Habelitz and B. J. Rodriguez, Piezoelectric properties of aligned collagen membranes, $J$. Biomed. Mater. Res., Part B, 2014, 102(2), 284-292.

98 Z. Zhou, D. Qian and M. Minary-Jolandan, Molecular Mechanism of Polarization and Piezoelectric Effect in Super-Twisted Collagen, ACS Biomater. Sci. Eng., 2016, 2(6), 929-936.

99 A. Kholkin, N. Amdursky, I. Bdikin, E. Gazit and G. Rosenman, Strong piezoelectricity in bioinspired peptide nanotubes, ACS Nano, 2010, 4(2), 610-614.

100 V. D. Parvanova and M. M. Nadoliisky, Polarization processes in PZT ceramics, Bulg. J. Phys., 2005, 32, 45-50. 
101 S. N. Cha, J. S. Seo, S. M. Kim, H. J. Kim, Y. J. Park, S. W. Kim and J. M. Kim, Sound-driven piezoelectric nanowire-based nanogenerators, Adv. Mater., 2010, 22(42), 4726-4730.

102 S. Bettini, R. Pagano, V. Bonfrate, E. Maglie, D. Manno, A. Serra, L. Valli and G. Giancane, Promising Piezoelectric Properties of New ZnO@Octadecylamine Adduct, J. Phys. Chem. C, 2015, 119(34), 20143-20149.

103 D. Choi, M. Y. Choi, W. M. Choi, H. J. Shin, H. K. Park, J. S. Seo, J. Park, S. M. Yoon, S. J. Chae, Y. H. Lee and S. W. Kim, Fully rollable transparent nanogenerators based on graphene electrodes, Adv. Mater., 2010, 22(19), 2187-2192.

104 M. Y. Lu, J. Song, M. P. Lu, C. Y. Lee, L. J. Chen and Z. L. Wang, ZnO-ZnS heterojunction and $\mathrm{ZnS}$ nanowire arrays for electricity generation, ACS Nano, 2009, 3(2), 357-362.

105 Q. Liao, Z. Zhang, X. Zhang, M. Mohr, Y. Zhang and H. J. Fecht, Flexible piezoelectric nanogenerators based on a fiber/ZnO nanowires/paper hybrid structure for energy harvesting, Nano Res., 2014, 7(6), 917-928.

106 G. Zhu, A. C. Wang, Y. Liu, Y. Zhou and Z. L. Wang, Functional electrical stimulation by nanogenerator with 58 V output voltage, Nano Lett., 2012, 12(6), 3086-3090.

107 S. Stassi, V. Cauda, C. Ottone, A. Chiodoni, C. F. Pirri and G. Canavese, Flexible piezoelectric energy nanogenerator based on $\mathrm{ZnO}$ nanotubes hosted in a polycarbonate membrane, Nano Energy, 2015, 13, 474-481.

108 J. Zhou, N. S. Xu and Z. L. Wang, Dissolving behavior and stability of $\mathrm{ZnO}$ wires in biofluids: a study on biodegradability and biocompatibility of $\mathrm{ZnO}$ nanostructures, Adv. Mater., 2006, 18(18), 2432-2435.

109 Z. G. Zhu, B. S. Li, G. R. Li, W. Z. Zhang and Q. R. Yin, Microstructure and piezoelectric properties of PMSPZT ceramics, Mater. Sci. Eng., B, 2005, 117(2), 216220.

110 S. Yang, L. Wang, X. Tian, Z. Xu, W. Wang, X. Bai and E. Wang, The piezotronic effect of zinc oxide nanowires studied by in situ TEM, Adv. Mater., 2012, 24(34), 46764682 .

111 B. Kumar and S. W. Kim, Energy harvesting based on semiconducting piezoelectric ZnO nanostructures, Nano Energy, 2012, 1(3), 342-355.

112 B. Zhao, N. Wang and G. Jiang, Controllable assembly and properties of $\mathrm{ZnO}$ nanowires, Microelectron. Int., 2015, 32(2), 52-54.

113 Z. L. Wang, G. Zhu, Y. Yang, S. Wang and C. Pan, Progress in nanogenerators for portable electronics, Mater. Today, 2012, 15(12), 532-543.

114 J. D. Major, R. Tena-Zaera, E. Azaceta, L. Bowen and K. Durose, Development of $\mathrm{ZnO}$ nanowire based CdTe thin film solar cells, Sol. Energy Mater. Sol. Cells, 2017, 160, 107-115.

115 P. V. Bakharev and D. N. McIlroy, The effect of the periodic boundary conditions of a ZnO-coated nanospring on its surface redox-induced electrical response, Nanotechnology, 2014, 25(47), 475501.
116 M. Kaur, S. Kailasaganapathi, N. Ramgir, N. Datta, S. Kumar, A. K. Debnath, D. K. Aswal and S. K. Gupta, Gas dependent sensing mechanism in $\mathrm{ZnO}$ nanobelt sensor, Appl. Surf. Sci., 2017, 394, 258-266.

117 V. Sahu, S. Goel, R. K. Sharma and G. Singh, Zinc oxide nanoring embedded lacey graphene nanoribbons in symmetric/asymmetric electrochemical capacitive energy storage, Nanoscale, 2015, 7(48), 20642-20651.

118 J. Zhou, P. Fei, Y. Gu, W. Mai, Y. Gao, R. Yang, G. Bao and Z. L. Wang, Piezoelectric-potential-controlled polarityreversible Schottky diodes and switches of $\mathrm{ZnO}$ wires, Nano Lett., 2008, 8(11), 3973-3977.

119 Z. Gao, Y. Ding, S. Lin, Y. Hao and Z. L. Wang, Dynamic fatigue studies of $\mathrm{ZnO}$ nanowires by in situ transmission electron microscopy, Phys. Status Solidi RRL, 2009, 3(7-8), 260-262.

120 M. H. Huang, S. Mao, H. Feick, H. Yan, Y. Wu, H. Kind, E. Weber, R. Russo and P. Yang, Room-temperature ultraviolet nanowire nanolasers, Science, 2001, 292(5523), 1897-1899.

121 J. Zhou, Y. Gu, Y. Hu, W. Mai, P. H. Yeh, G. Bao, A. K. Sood, D. L. Polla and Z. L. Wang, Gigantic enhancement in response and reset time of $\mathrm{ZnO}$ UV nanosensor by utilizing Schottky contact and surface functionalization, Appl. Phys. Lett., 2009, 94(19), 191103.

122 X. W. Sun, J. Z. Huang, J. X. Wang and Z. Xu, A ZnO nanorod inorganic/organic heterostructure light-emitting diode emitting at 342 nm, Nano Lett., 2008, 8(4), 1219-1223.

123 E. Comini, G. Faglia, G. Sberveglieri, Z. Pan and Z. L. Wang, Stable and highly sensitive gas sensors based on semiconducting oxide nanobelts, Appl. Phys. Lett., 2002, 81(10), 1869-1871.

124 M. Saito and S. Fujihara, Large photocurrent generation in dye-sensitized ZnO solar cells, Energy Environ. Sci., 2008, 1(2), 280-283.

125 S. H. Shin, Y. H. Kim, M. H. Lee, J. Y. Jung and J. Nah, Hemispherically aggregated $\mathrm{BaTiO}_{3}$ nanoparticle composite thin film for high-performance flexible piezoelectric nanogenerator, ACS Nano, 2014, 8(3), 27662773.

126 C. K. Jeong, I. Kim, K. I. Park, M. H. Oh, H. Paik, G. T. Hwang, K. No, Y. S. Nam and K. J. Lee, Virusdirected design of a flexible $\mathrm{BaTiO}_{3}$ nanogenerator, ACS Nano, 2013, 7(12), 11016-11025.

127 C. Y. Chen, T. H. Liu, Y. Zhou, Y. Zhang, Y. L. Chueh, Y. H. Chu, J. H. He and Z. L. Wang, Electricity generation based on vertically aligned $\mathrm{PbZr}_{0.2} \mathrm{Ti}_{0.8} \mathrm{O}_{3}$ nanowire arrays, Nano Energy, 2012, 1(3), 424-428.

128 M. H. Zhao, Z. L. Wang and S. X. Mao, Piezoelectric characterization of individual zinc oxide nanobelt probed by piezoresponse force microscope, Nano Lett., 2004, 4(4), 587-590.

129 Z. L. Wang, Zinc oxide nanostructures: growth, properties and applications, J. Phys.: Condens. Matter, 2004, 16(25), R829.

130 N. Soin, T. H. Shah, S. C. Anand, J. Geng, W. Pornwannachai, P. Mandal, D. Reid, S. Sharma, 
R. L. Hadimani, D. V. Bayramol and E. Siores, Novel “3-D spacer" all fibre piezoelectric textiles for energy harvesting applications, Energy Environ. Sci., 2014, 7(5), 1670-1679.

131 W. Zeng, X. M. Tao, S. Chen, S. Shang, H. L. Chan and S. H. Choy, Highly durable all-fiber nanogenerator for mechanical energy harvesting, Energy Environ. Sci., 2013, 6(9), 2631-2638.

132 J. H. Lee, K. Y. Lee, B. Kumar, N. T. Tien, N. E. Lee and S. W. Kim, Highly sensitive stretchable transparent piezoelectric nanogenerators, Energy Environ. Sci., 2013, 6(1), 169-175.

133 Y. Bin, Y. Hao, M. Mengye, W. Hongzhi and Z. Meifang, Enhanced output power polyvinylidene fluoride (PVDF) electrospun nanogenerator with high fiber alignment, in Nanotechnology (IEEE-NANO), 2015 IEEE 15th International Conference on IEEE, 2015, pp. 932-935.

134 J. Liu, N. Cui, L. Gu, X. Chen, S. Bai, Y. Zheng, C. Hu and Y. Qin, A three-dimensional integrated nanogenerator for effectively harvesting sound energy from the environment, Nanoscale, 2016, 8(9), 4938-4944.

135 M. S. Bafqi, R. Bagherzadeh and M. Latifi, Fabrication of composite PVDF-ZnO nanofiber mats by electrospinning for energy scavenging application with enhanced efficiency, J. Polym. Res., 2015, 22(7), 130.

136 L. Persano, C. Dagdeviren, Y. Su, Y. Zhang, S. Girardo, D. Pisignano, Y. Huang and J. A. Rogers, High performance piezoelectric devices based on aligned arrays of nanofibers of poly(vinylidenefluoride-co-trifluoroethylene), Nat. Commun., 2013, 4, 1633.

137 P. Martins, C. Caparros, R. Gonçalves, P. M. Martins, M. Benelmekki, G. Botelho and S. Lanceros-Mendez, Role of nanoparticle surface charge on the nucleation of the electroactive $\beta$-poly(vinylidene fluoride) nanocomposites for sensor and actuator applications, J. Phys. Chem. C, 2012, 116(29), 15790-15794.

138 C. Chang, V. H. Tran, J. Wang, Y. K. Fuh and L. Lin, Directwrite piezoelectric polymeric nanogenerator with high energy conversion efficiency, Nano Lett., 2010, 10(2), 726731.

139 D. Y. Lee, H. Kim, H. M. Li, A. R. Jang, Y. D. Lim, S. N. Cha, Y. J. Park, D. J. Kang and W. J. Yoo, Hybrid energy harvester based on nanopillar solar cells and PVDF nanogenerator, Nanotechnology, 2013, 24(17), 175402.

140 S. Cha, S. M. Kim, H. Kim, J. Ku, J. I. Sohn, Y. J. Park, B. G. Song, M. H. Jung, E. K. Lee, B. L. Choi and J. J. Park, Porous PVDF as effective sonic wave driven nanogenerators, Nano Lett., 2011, 11(12), 5142-5147.

141 D. Dhakras, V. Borkar, S. Ogale and J. Jog, Enhanced piezoresponse of electrospun PVDF mats with a touch of nickel chloride hexahydrate salt, Nanoscale, 2012, 4(3), 752-756.

142 C. Sun, J. Shi, D. J. Bayerl and X. Wang, PVDF microbelts for harvesting energy from respiration, Energy Environ. Sci., 2011, 4(11), 4508-4512.

143 J. Fang, X. Wang and T. Lin, Electrical power generator from randomly oriented electrospun poly(vinylidene fluoride) nanofibre membranes, J. Mater. Chem., 2011, 21(30), 11088-11091.

144 Y. J. Hsu, S. Y. Lu and Y. F. Lin, One-Step Preparation of Coaxial CdS-ZnS and $\mathrm{Cd}_{1-x} \mathrm{Zn}_{x} \mathrm{~S}-\mathrm{ZnS}$ Nanowires, Adv. Funct. Mater., 2005, 15(8), 1350-1357.

145 Q. H. Li, T. Gao and T. H. Wang, Optoelectronic characteristics of single CdS nanobelts, Appl. Phys. Lett., 2005, 86(19), 193109.

146 A. R. Hutson, Piezoelectricity and conductivity in ZnO and CdS, Phys. Rev. Lett., 1960, 4(10), 505.

147 Y. F. Lin, J. Song, Y. Ding, S. Y. Lu and Z. L. Wang, Piezoelectric nanogenerator using CdS nanowires, Appl. Phys. Lett., 2008, 92(2), 022105.

148 Y. J. Hsu and S. Y. Lu, Photoluminescence resulting from semiconductor-metal solid solution observed in onedimensional semiconductor nanostructures, Langmuir, 2004, 20(1), 23-26.

149 J. Zhang, F. Jiang and L. Zhang, Fabrication of singlecrystalline semiconductor CdS nanobelts by vapor transport, J. Phys. Chem. B, 2004, 108(22), 7002-7005.

150 Y. F. Lin, Y. J. Hsu, S. Y. Lu and S. C. Kung, Non-catalytic and template-free growth of aligned $\mathrm{CdS}$ nanowires exhibiting high field emission current densities, Chem. Commun., 2006, (22), 2391-2393.

151 X. P. Shen, A. H. Yuan, F. Wang, J. M. Hong and Z. Xu, Fabrication of well-aligned CdS nanotubes by CVDtemplate method, Solid State Commun., 2005, 133(1), 19-22.

152 Y. Huang, X. Duan, Y. Cui and C. M. Lieber, Gallium nitride nanowire nanodevices, Nano Lett., 2002, 2(2), 101-104.

153 T. Kuykendall, P. Pauzauskie, S. Lee, Y. Zhang, J. Goldberger and P. Yang, Metalorganic chemical vapor deposition route to GaN nanowires with triangular cross sections, Nano Lett., 2003, 3(8), 1063-1066.

154 C. T. Huang, J. Song, W. F. Lee, Y. Ding, Z. Gao, Y. Hao, L. J. Chen and Z. L. Wang, GaN nanowire arrays for highoutput nanogenerators, J. Am. Chem. Soc., 2010, 132(13), 4766-4771.

155 L. Lin, C. H. Lai, Y. Hu, Y. Zhang, X. Wang, C. Xu, R. L. Snyder, L. J. Chen and Z. L. Wang, High output nanogenerator based on assembly of GaN nanowires, Nanotechnology, 2011, 22(47), 475401.

156 K. Yin, H. Lin, Q. Cai, Y. Zhao, S. T. Lee, F. Hu and M. Shao, Silicon nanowires nanogenerator based on the piezoelectricity of alpha-quartz, Nanoscale, 2013, 5(24), 12330-12334.

157 Label PI, oHS Compliance Engineer R. Directive 2002/95/ EC of the European Parliament and of the Council of 27 January 2003 on the restriction of the use of certain hazardous substances in electrical and electronic equipment.

158 W. Wu, S. Bai, M. Yuan, Y. Qin, Z. L. Wang and T. Jing, Lead zirconate titanate nanowire textile nanogenerator for wearable energy-harvesting and self-powered devices, ACS Nano, 2012, 6(7), 6231-6235. 
159 S. Xu, G. Poirier and N. Yao, PMN-PT nanowires with a very high piezoelectric constant, Nano Lett., 2012, 12(5), 22382242.

160 S. Xu, Y. W. Yeh, G. Poirier, M. C. McAlpine, R. A. Register and N. Yao, Flexible piezoelectric PMN-PT nanowire-based nanocomposite and device, Nano Lett., 2013, 13(6), 23932398.

161 F. Wu, W. Cai, Y. W. Yeh, S. Xu and N. Yao, Energy scavenging based on a single-crystal PMN-PT nanobelt, Sci. Rep., 2016, 6, 22513.

162 G. Zhang, Q. Liao, Z. Zhang, Q. Liang, Y. Zhao, X. Zheng and Y. Zhang, Novel Piezoelectric Paper-Based Flexible Nanogenerators Composed of $\mathrm{BaTiO}_{3}$ Nanoparticles and Bacterial Cellulose, Adv. Sci., 2016, 3(2), 1500257.

163 Z. Wang, J. Hu, A. P. Suryavanshi, K. Yum and M. F. Yu, Voltage generation from individual $\mathrm{BaTiO}_{3}$ nanowires under periodic tensile mechanical load, Nano Lett., 2007, 7(10), 2966-2969.

164 K. I. Park, M. Lee, Y. Liu, S. Moon, G. T. Hwang, G. Zhu, J. E. Kim, S. O. Kim, D. K. Kim, Z. L. Wang and K. J. Lee, Flexible nanocomposite generator made of $\mathrm{BaTiO}_{3}$ nanoparticles and graphitic carbons, Adv. Mater., 2012, 24(22), 2999-3004.

165 Z. H. Lin, Y. Yang, J. M. Wu, Y. Liu, F. Zhang and Z. L. Wang, $\mathrm{BaTiO}_{3}$ nanotubes-based flexible and transparent nanogenerators, J. Phys. Chem. Lett., 2012, 3(23), 3599-3604.

166 K. I. Park, S. Xu, Y. Liu, G. T. Hwang, S. J. Kang, Z. L. Wang and K. J. Lee, Piezoelectric $\mathrm{BaTiO}_{3}$ thin film nanogenerator on plastic substrates, Nano Lett., 2010, 10(12), 4939-4943.

167 T. Y. Ke, H. A. Chen, H. S. Sheu, J. W. Yeh, H. N. Lin, C. Y. Lee and H. T. Chiu, Sodium niobate nanowire and its piezoelectricity, J. Phys. Chem. C, 2008, 112(24), 88278831.

168 Y. Yang, J. H. Jung, B. K. Yun, F. Zhang, K. C. Pradel, W. Guo and Z. L. Wang, Flexible Pyroelectric Nanogenerators using a Composite Structure of Lead-Free $\mathrm{KNbO}_{3}$ Nanowires, $A d v$. Mater., 2012, 24(39), 5357-5362.

169 J. H. Jung, C. Y. Chen, B. K. Yun, N. Lee, Y. Zhou, W. Jo, L. J. Chou and Z. L. Wang, Lead-free $\mathrm{KNbO}_{3}$ ferroelectric nanorod based flexible nanogenerators and capacitors, Nanotechnology, 2012, 23(37), 375401.

170 M. R. Joung, H. Xu, I. T. Seo, D. H. Kim, J. Hur, S. Nahm, C. Y. Kang, S. J. Yoon and H. M. Park, Piezoelectric nanogenerators synthesized using $\mathrm{KNbO}_{3}$ nanowires with various crystal structures, J. Mater. Chem. A, 2014, 2(43), 18547-18553.

171 A. Fujishima, T. N. Rao and D. A. Tryk, Titanium dioxide photocatalysis, J. Photochem. Photobiol., C, 2000, 1(1), 121.

172 K. Rajeshwar and J. G. Ibanez, Electrochemical aspects of photocatalysis: application to detoxification and disinfection scenarios, J. Chem. Educ., 1995, 72(11), 1044.

173 D. A. Tryk, A. Fujishima and K. Honda, Recent topics in photoelectrochemistry: achievements and future prospects, Electrochim. Acta, 2000, 45(15), 2363-2376.
174 R. Paily, A. DasGupta, N. DasGupta, P. Bhattacharya, P. Misra, T. Ganguli, L. M. Kukreja, A. K. Balamurugan, S. Rajagopalan and A. K. Tyagi, Pulsed laser deposition of $\mathrm{TiO}_{2}$ for MOS gate dielectric, Appl. Surf. Sci., 2002, 187(3), 297-304.

175 (a) P. Kajitvichyanukul, J. Ananpattarachai and S. Pongpom, Sol-gel preparation and properties study of $\mathrm{TiO}_{2}$ thin film for photocatalytic reduction of chromium(VI) in photocatalysis process, Sci. Technol. Adv. Mater., 2005, 6(3), 352-358; (b) P. Paunović, A. Češnovar, A. Grozdanov, P. Makreski and E. Fidančevska, Preparation of nano-crystalline $\mathrm{TiO}_{2}$ by sol-gel method using titanium tetraisopropoxide (TTIP) as a precursor, Adv. Nat. Sci., 2012, 1(2), 133-142.

176 F. Rubio-Marcos, P. Ochoa and J. F. Fernandez, Sintering and properties of lead-free (K,Na,Li)(Nb,Ta,Sb) $\mathrm{O}_{3}$ ceramics, J. Eur. Ceram. Soc., 2007, 27(13), 4125-4129.

177 F. Rubio-Marcos, A. Del Campo, R. López-Juárez, J. J. Romero and J. F. Fernández, High spatial resolution structure of $(\mathrm{K}, \mathrm{Na}) \mathrm{NbO}_{3}$ lead-free ferroelectric domains, J. Mater. Chem., 2012, 22(19), 9714-9720.

178 F. Rubio-Marcos, R. López-Juárez, R. E. Rojas-Hernandez, A. del Campo, N. Razo-Pérez and J. F. Fernandez, Lead-free piezoceramics: revealing the role of the rhombohedraltetragonal phase coexistence in enhancement of the piezoelectric properties, ACS Appl. Mater. Interfaces, 2015, 7(41), 23080-23088.

179 K. N. Kim, J. Chun, S. A. Chae, C. W. Ahn, I. W. Kim, S. W. Kim, Z. L. Wang and J. M. Baik, Silk fibroin-based biodegradable piezoelectric composite nanogenerators using lead-free ferroelectric nanoparticles, Nano Energy, 2015, 14, 87-94.

180 G. Shirane, R. Newnham and R. Pepinsky, Dielectric Properties and Phase Transitions of $\mathrm{NaNbO}_{3}$ and $(\mathrm{Na}, \mathrm{K})$ $\mathrm{NbO}_{3}$, Phys. Rev., 1954, 96(3), 581.

181 L. Egerton and D. M. Dillon, Piezoelectric and dielectric properties of ceramics in the system potassium-sodium niobate, J. Am. Ceram. Soc., 1959, 42(9), 438-442.

182 R. E. Jaeger and L. Egerton, Hot Pressing of PotassiumSodium Niobates, J. Am. Ceram. Soc., 1962, 45(5), 209-213.

183 H. B. Kang, J. Chang, K. Koh, L. Lin and Y. S. Cho, High quality Mn-doped (Na,K) $\mathrm{NbO}_{3}$ nanofibers for flexible piezoelectric nanogenerators, ACS Appl. Mater. Interfaces, 2014, 6(13), 10576-10582.

184 B. Y. Kim, I. T. Seo, Y. S. Lee, J. S. Kim, S. Nahm, C. Y. Kang, S. J. Yoon, J. H. Paik and Y. H. Jeong, High-Performance $\left(\mathrm{Na}_{0.5} \mathrm{~K}_{0.5}\right) \mathrm{NbO}_{3}$ Thin Film Piezoelectric Energy Harvester, J. Am. Ceram. Soc., 2015, 98(1), 119-124.

185 C. K. Jeong, K. I. Park, J. Ryu, G. T. Hwang and K. J. Lee, Large-Area and Flexible Lead-Free Nanocomposite Generator Using Alkaline Niobate Particles and Metal Nanorod Filler, Adv. Funct. Mater., 2014, 24(18), 2620-2629.

186 F. Wang, C. Jiang, C. Tang, S. Bi, Q. Wang, D. Du and J. Song, High output nano-energy cell with piezoelectric nanogenerator and porous supercapacitor dual functionsA technique to provide sustaining power by harvesting 
intermittent mechanical energy from surroundings, Nano Energy, 2016, 21, 209-216.

187 H. Xu, M. R. Joung, J. S. Kim, S. Nahm, M. G. Kang, C. Y. Kang and S. J. Yoon, Synthesis of homogeneous $\left(\mathrm{Na}_{1-x} \mathrm{~K}_{x}\right) \mathrm{NbO}_{3}$ nanorods using hydrothermal and postheat treatment processes, Chem. Eng. J., 2012, 211, 16-21.

188 J. Wu, D. Xiao, W. Wu, Q. Chen, J. Zhu, Z. Yang and J. Wang, Role of room-temperature phase transition in the electrical properties of $(\mathrm{Ba}, \mathrm{Ca})(\mathrm{Ti}, \mathrm{Zr}) \mathrm{O}_{3}$ ceramics, Scr. Mater., 2011, 65(9), 771-774.

189 S. B. Li, L. M. Zhang, C. B. Wang, X. Ji and Q. Shen, Structural, dielectric and ferroelectric properties of leadfree $\mathrm{Ba}_{0.85} \mathrm{Ca}_{0.15} \mathrm{Zr}_{0.10} \mathrm{Ti}_{0.90} \mathrm{O}_{3}$ ceramics prepared by Plasma Activated Sintering, Ceram. Int., 2016, 42(16), 18585-18591.

190 Y. Kou, Z. Kou, D. Zhao, Z. Wang, G. Gao and X. Chai, Fabrication of lead-free $\mathrm{Ba}\left(\mathrm{Zr}_{0.2} \mathrm{Ti}_{0.8}\right) \mathrm{O}_{3}-\left(\mathrm{Ba}_{0.7} \mathrm{Ca}_{0.3}\right) \mathrm{TiO}_{3}$ nanoparticles and the application in flexible piezoelectric nanogenerator, Ceram. Int., 2017, 43(6), 4803-4806.

191 Y. Lai, Y. Zeng, X. Tang, H. Zhang, J. Han, Z. Huang and $\mathrm{H}$. Su, Effects of $\mathrm{CaO}-\mathrm{B}_{2} \mathrm{O}_{3}-\mathrm{SiO}_{2}$ glass additive on the microstructure and electrical properties of BCZT lead-free ceramic, Ceram. Int., 2016, 42(11), 12694-12700.

192 J. Wu, D. Xiao, W. Wu, Q. Chen, J. Zhu, Z. Yang and J. Wang, Composition and poling condition-induced electrical behavior of $\left(\mathrm{Ba}_{0.85} \mathrm{Ca}_{0.15}\right)\left(\mathrm{Ti}_{1-x} \mathrm{Zr}_{x}\right) \mathrm{O}_{3}$ lead-free piezoelectric ceramics, J. Eur. Ceram. Soc., 2012, 32(4), 891-898.

$193 \mathrm{~W}$. Liu and X. Ren, Large piezoelectric effect in Pb-free ceramics, Phys. Rev. Lett., 2009, 103(25), 257602.

194 Y. Kou, X. Chai, R. Yu, Y. Liu and Z. Wang, Bio-compatible BCTZ-based piezoelectric nanogenerator as energy harvester and waterdrop counter, Ceram. Int., 2017, 43(9), 6666-6670.

195 Z. Wang, J. Liu, F. Wang, S. Chen, H. Luo and X. Yu, Sizecontrolled synthesis of $\mathrm{ZnSnO}_{3}$ cubic crystallites at low temperatures and their HCHO-sensing properties, J. Phys. Chem. C, 2010, 114(32), 13577-13582.

196 J. Zhang, K. L. Yao, Z. L. Liu, G. Y. Gao, Z. Y. Sun and S. W. Fan, First-principles study of the ferroelectric and nonlinear optical properties of the $\mathrm{LiNbO}_{3}$-type $\mathrm{ZnSnO}_{3}$, Phys. Chem. Chem. Phys., 2010, 12(32), 9197-9204.

197 H. Gou, F. Gao and J. Zhang, Structural identification, electronic and optical properties of $\mathrm{ZnSnO}_{3}$ : first principle calculations, Comput. Mater. Sci., 2010, 49(3), 552-555.

198 H. Wang, H. Huang and B. Wang, First-principles study of structural, electronic, and optical properties of, Solid State Commun., 2009, 149(41), 1849-1852.

199 Z. Tian, C. Liang, J. Liu, H. Zhang and L. Zhang, Zinc stannate nanocubes and nanourchins with high photocatalytic activity for methyl orange and 2,5-DCP degradation, J. Mater. Chem., 2012, 22(33), 17210-17214.

200 K. Y. Lee, D. Kim, J. H. Lee, T. Y. Kim, M. K. Gupta and S. W. Kim, Unidirectional High-Power Generation via Stress-Induced Dipole Alignment from $\mathrm{ZnSnO}_{3}$
Nanocubes/Polymer Hybrid Piezoelectric Nanogenerator, Adv. Funct. Mater., 2014, 24(1), 37-43.

201 Y. Zeng, T. Zhang, H. Fan, W. Fu, G. Lu, Y. Sui and H. Yang, One-pot synthesis and gas-sensing properties of hierarchical $\mathrm{ZnSnO}_{3}$ nanocages, J. Phys. Chem. C, 2009, 113(44), 19000-19004.

202 P. Song, Q. Wang and Z. Yang, Biomorphic synthesis of $\mathrm{ZnSnO}_{3}$ hollow fibers for gas sensing application, Sens. Actuators, B, 2011, 156(2), 983-989.

203 S. Suyitno, A. Purwanto, R. L. Hidayat, I. Sholahudin, M. Yusuf, S. Huda and Z. Arifin, Fabrication and Characterization of Zinc Oxide-Based Electrospun Nanofibers for Mechanical Energy Harvesting, $J$. Nanotechnol. Eng. Med., 2014, 5(1), 011002.

204 M. A. Kanjwal, F. A. Sheikh, N. A. Barakat, X. Li, H. Y. Kim and I. S. Chronakis, $\mathrm{Co}_{3} \mathrm{O}_{4}, \mathrm{ZnO}, \mathrm{Co}_{3} \mathrm{O}_{4}-\mathrm{ZnO}$ Nanofibers and Their Properties, J. Nanoeng. Nanomanuf., 2011, 1(2), 196-202.

205 D. Subagiyo, M. Thoyib, H. S. Suyitno, A. Jamaluddin and R. L. Hidayat, Effect of codoping cobalt and aluminum on enhancing the piezoelectricity properties of fiber-based zinc oxide, in AIP Conference Proceedings, ed. B. Kristiawan, M. Anwar, A. T. Wijayanta, S. Hadi and D. Danardono, 2017, AIP Publishing, vol. 1788, no. 1, p. 030059.

206 H. Xu, T. G. Lee, S. J. Park, B. Y. Kim and S. Nahm, Sodiumpotassium niobate nanorods with various crystal structures and their application to nanogenerator, J. Am. Ceram. Soc., 2017, 100(4), 1673-1681.

207 H. Im, T. Kim, H. Song, J. Choi, J. S. Park, R. Ovalle-Robles, H. D. Yang, K. D. Kihm, R. H. Baughman, H. H. Lee and T. J. Kang, High-efficiency electrochemical thermal energy harvester using carbon nanotube aerogel sheet electrodes, Nat. Commun., 2016, 7, 10600.

208 C. Y. Chiang, Y. Shin and S. Ehrman, Li doped CuO film electrodes for photoelectrochemical cells, J. Electrochem. Soc., 2011, 159(2), B227-B231.

209 M. A. Rahman, B. C. Lee, D. T. Phan and G. S. Chung, Fabrication and characterization of highly efficient flexible energy harvesters using PVDF-graphene nanocomposites, Smart Mater. Struct., 2013, 22(8), 085017.

210 J. Xue, L. Wu, N. Hu, J. Qiu, C. Chang, S. Atobe, H. Fukunaga, T. Watanabe, Y. Liu, H. Ning and J. Li, Evaluation of piezoelectric property of reduced graphene oxide (rGO)-poly(vinylidene fluoride) nanocomposites, Nanoscale, 2012, 4(22), 7250-7255.

211 J. S. Lee, K. Y. Shin, C. Kim and J. Jang, Enhanced frequency response of a highly transparent PVDF-graphene based thin film acoustic actuator, Chem. Commun., 2013, 49(94), 11047-11049.

212 L. Wu, Alamusi, J. Xue, T. Itoi, N. Hu, Y. Li, C. Yan, J. Qiu, H. Ning, W. Yuan and B. Gu, Improved energy harvesting capability of poly(vinylidene fluoride) films modified by reduced graphene oxide, J. Intell. Mater. Syst. Struct., 2014, 25(14), 1813-1824.

213 S. Ansari and E. P. Giannelis, Functionalized graphene sheet-poly(vinylidene fluoride) conductive 
nanocomposites, J. Polym. Sci., Part B: Polym. Phys., 2009, 47(9), 888-897.

214 R. K. Layek, S. Samanta, D. P. Chatterjee and A. K. Nandi, Physical and mechanical properties of poly(methyl methacrylate)-functionalized graphene/poly(vinylidine fluoride) nanocomposites: Piezoelectric $\beta$ polymorph formation, Polymer, 2010, 51(24), 5846-5856.

215 C. M. Wu and M. H. Chou, Sound absorption of electrospun polyvinylidene fluoride/graphene membranes, Eur. Polym. J., 2016, 82, 35-45.

216 Y. Hu, Y. Liu, W. Li, M. Gao, X. Liang, Q. Li and L. M. Peng, Observation of a 2D Electron Gas and the Tuning of the Electrical Conductance of ZnO Nanowires by Controllable Surface Band-Bending, Adv. Funct. Mater., 2009, 19(15), 2380-2387.

217 S. Lu, J. Qi, Y. Gu, S. Liu, Q. Xu, Z. Wang, Q. Liang and Y. Zhang, Influence of the carrier concentration on the piezotronic effect in a $\mathrm{ZnO} / \mathrm{Au}$ Schottky junction, Nanoscale, 2015, 7(10), 4461-4467.

218 Y. Hu, L. Lin, Y. Zhang and Z. L. Wang, Replacing a battery by a nanogenerator with $20 \mathrm{~V}$ output, Adv. Mater., 2012, 24(1), 110-114.

219 T. T. Pham, K. Y. Lee, J. H. Lee, K. H. Kim, K. S. Shin, M. K. Gupta, B. Kumar and S. W. Kim, Reliable operation of a nanogenerator under ultraviolet light via engineering piezoelectric potential, Energy Environ. Sci., 2013, 6(3), 841-846.

220 J. I. Sohn, S. N. Cha, B. G. Song, S. Lee, S. M. Kim, J. Ku, H. J. Kim, Y. J. Park, B. L. Choi, Z. L. Wang and J. M. Kim, Engineering of efficiency limiting free carriers and an interfacial energy barrier for an enhancing piezoelectric generation, Energy Environ. Sci., 2013, 6(1), 97-104.

221 S. Lee, J. Lee, W. Ko, S. Cha, J. Sohn, J. Kim, J. Park, Y. Park and J. Hong, Solution-processed Ag-doped $\mathrm{ZnO}$ nanowires grown on flexible polyester for nanogenerator applications, Nanoscale, 2013, 5(20), 9609-9614.

222 S. H. Shin, Y. H. Kim, M. H. Lee, J. Y. Jung, J. H. Seol and J. Nah, Lithium-Doped Zinc Oxide Nanowires-Polymer Composite for High Performance Flexible Piezoelectric Nanogenerator, ACS Nano, 2014, 8(10), 10844-10850.

223 J. Song, X. Wang, J. Liu, H. Liu, Y. Li and Z. L. Wang, Piezoelectric potential output from $\mathrm{ZnO}$ nanowire functionalized with p-type oligomer, Nano Lett., 2008, 8(1), 203-207.

224 Y. H. Kwon, D. H. Kim, H. K. Kim and J. Nah, Phosphorusdoped zinc oxide $\mathrm{p}-\mathrm{n}$ homojunction thin film for flexible piezoelectric nanogenerators, Nano Energy, 2015, 18, 126132.

225 S. H. Shin, M. H. Lee, J. Y. Jung, J. H. Seol and J. Nah, Piezoelectric performance enhancement of $\mathrm{ZnO}$ flexible nanogenerator by a $\mathrm{CuO}-\mathrm{ZnO} \mathrm{p}-\mathrm{n}$ junction formation, $J$. Mater. Chem. C, 2013, 1(48), 8103-8107.

226 K. Y. Lee, B. Kumar, J. S. Seo, K. H. Kim, J. I. Sohn, S. N. Cha, D. Choi, Z. L. Wang and S. W. Kim, P-Type polymerhybridized high-performance piezoelectric nanogenerators, Nano Lett., 2012, 12(4), 1959-1964.
227 K. Y. Lee, J. Bae, S. Kim, J. H. Lee, G. C. Yoon, M. K. Gupta, S. Kim, H. Kim, J. Park and S. W. Kim, Depletion width engineering via surface modification for high performance semiconducting piezoelectric nanogenerators, Nano Energy, 2014, 8, 165-173.

228 B. Yin, Y. Qiu, H. Zhang, J. Lei, Y. Chang, J. Ji, Y. Luo, Y. Zhao and L. Hu, Piezoelectric performance enhancement of $\mathrm{ZnO}$ flexible nanogenerator by a NiOZnO p-n junction formation, Nano Energy, 2015, 14, 95101.

229 J. Briscoe, M. Stewart, M. Vopson, M. Cain, P. M. Weaver and S. Dunn, Nanostructured p-n Junctions for Kineticto-Electrical Energy Conversion, Adv. Energy Mater., 2012, 2(10), 1261-1268.

230 M. R. Hasan, S. H. Baek, K. S. Seong, J. H. Kim and I. K. Park, Hierarchical ZnO nanorods on Si micropillar arrays for performance enhancement of piezoelectric nanogenerators, ACS Appl. Mater. Interfaces, 2015, 7(10), 5768-5774.

231 S. Lu, Q. Liao, J. Qi, S. Liu, Y. Liu, Q. Liang, G. Zhang and Y. Zhang, The enhanced performance of piezoelectric nanogenerator via suppressing screening effect with $\mathrm{Au}$ particles/ZnO nanoarrays Schottky junction, Nano Res., 2016, 9(2), 372-379.

232 Y. F. Lin, J. Song, Y. Ding, S. Y. Lu and Z. L. Wang, Alternating the output of a CdS nanowire nanogenerator by a white-light-stimulated optoelectronic effect, Adv. Mater., 2008, 20(16), 3127-3130.

233 K. C. Pradel, W. Wu, Y. Zhou, X. Wen, Y. Ding and Z. L. Wang, Piezotronic effect in solution-grown p-type ZnO nanowires and films, Nano Lett., 2013, 13(6), 26472653.

234 T. Li, Z. Huang, Z. Suo, S. P. Lacour and S. Wagner, Stretchability of thin metal films on elastomer substrates, Appl. Phys. Lett., 2004, 85(16), 3435-3437.

235 S. P. Lacour, D. Chan, S. Wagner, T. Li and Z. Suo, Mechanisms of reversible stretchability of thin metal films on elastomeric substrates, Appl. Phys. Lett., 2006, 88(20), 204103.

236 K. S. Kim, Y. Zhao, H. Jang, S. Y. Lee, J. M. Kim, K. S. Kim, J. H. Ahn, P. Kim, J. Y. Choi and B. H. Hong, Large-scale pattern growth of graphene films for stretchable transparent electrodes, Nature, 2009, 457(7230), 706-710.

237 L. Ci, J. Suhr, V. Pushparaj, X. Zhang and P. M. Ajayan, Continuous carbon nanotube reinforced composites, Nano Lett., 2008, 8(9), 2762-2766.

$238 \mathrm{Z}$. Yu, X. Niu, Z. Liu and Q. Pei, Intrinsically Stretchable Polymer Light-Emitting Devices Using Carbon NanotubePolymer Composite Electrodes, Adv. Mater., 2011, 23(34), 3989-3994.

239 Y. Y. Huang and E. M. Terentjev, Tailoring the electrical properties of carbon nanotube-polymer composites, Adv. Funct. Mater., 2010, 20(23), 4062-4068.

240 S. Ghosh, I. Calizo, D. Teweldebrhan, E. P. Pokatilov, D. L. Nika, A. A. Balandin, W. Bao, F. Miao and C. N. Lau, Extremely high thermal conductivity of graphene: Prospects for thermal management applications in 
nanoelectronic circuits, Appl. Phys. Lett., 2008, 92(15), 151911.

241 A. A. Balandin, S. Ghosh, W. Bao, I. Calizo, D. Teweldebrhan, F. Miao and C. N. Lau, Superior thermal conductivity of single-layer graphene, Nano Lett., 2008, 8(3), 902-907.

242 X. Li, Z. H. Lin, G. Cheng, X. Wen, Y. Liu, S. Niu and Z. L. Wang, 3D fiber-based hybrid nanogenerator for energy harvesting and as a self-powered pressure sensor, ACS Nano, 2014, 8(10), 10674-10681.

243 K. H. Kim, K. Y. Lee, J. S. Seo, B. Kumar and S. W. Kim, Paper-based piezoelectric nanogenerators with high thermal stability, Small, 2011, 7(18), 2577-2580.

244 C. K. Jeong, K. I. Park, J. H. Son, G. T. Hwang, S. H. Lee, D. Y. Park, H. E. Lee, H. K. Lee, M. Byun and K. J. Lee, Self-powered fully-flexible light-emitting system enabled by flexible energy harvester, Energy Environ. Sci., 2014, 7(12), 4035-4043.

245 B. J. Hansen, Y. Liu, R. Yang and Z. L. Wang, Hybrid nanogenerator for concurrently harvesting biomechanical and biochemical energy, ACS Nano, 2010, 4(7), 3647-3652.

246 J. Zhong, Y. Zhang, Q. Zhong, Q. Hu, B. Hu, Z. L. Wang and J. Zhou, Fiber-based generator for wearable electronics and mobile medication, ACS Nano, 2014, 8(6), 6273-6280.

247 L. Zhang, S. Bai, C. Su, Y. Zheng, Y. Qin, C. Xu and Z. L. Wang, A High-Reliability Kevlar Fiber-ZnO Nanowires Hybrid Nanogenerator and its Application on Self-Powered UV Detection, Adv. Funct. Mater., 2015, 25(36), 5794-5798.

248 M. Zhang, T. Gao, J. Wang, J. Liao, Y. Qiu, Q. Yang, H. Xue, Z. Shi, Y. Zhao, Z. Xiong and L. Chen, A hybrid fibers based wearable fabric piezoelectric nanogenerator for energy harvesting application, Nano Energy, 2015, 13, 298-305.

249 N. R. Alluri, B. Saravanakumar and S. J. Kim, Flexible, Hybrid Piezoelectric Film $\left(\mathrm{BaTi}_{(1-x)} \mathrm{Zr}_{x} \mathrm{O}_{3}\right) / \mathrm{PVDF}$ Nanogenerator as a Self-Powered Fluid Velocity Sensor, ACS Appl. Mater. Interfaces, 2015, 7(18), 9831-9840.

250 M. M. Alam, S. K. Ghosh, A. Sultana and D. Mandal, Leadfree $\mathrm{ZnSnO}_{3} / \mathrm{MWCNTs}$-based self-poled flexible hybrid nanogenerator for piezoelectric power generation, Nanotechnology, 2015, 26(16), 165403.

251 J. H. Lee, K. Y. Lee, M. K. Gupta, T. Y. Kim, D. Y. Lee, J. Oh, C. Ryu, W. J. Yoo, C. Y. Kang, S. J. Yoon and J. B. Yoo, Highly stretchable piezoelectric-pyroelectric hybrid nanogenerator, Adv. Mater., 2014, 26(5), 765-769.

252 X. Wang, B. Yang, J. Liu, Y. Zhu, C. Yang and Q. He, A flexible triboelectric-piezoelectric hybrid nanogenerator based on P(VDF-TrFE) nanofibers and PDMS/MWCNT for wearable devices, Sci. Rep., 2016, 6, 36409.

253 M. Han, X. S. Zhang, B. Meng, W. Liu, W. Tang, X. Sun, W. Wang and $\mathrm{H}$. Zhang, r-Shaped hybrid nanogenerator with enhanced piezoelectricity, ACS Nano, 2013, 7(10), 8554-8560.

254 Y. Zi, L. Lin, J. Wang, S. Wang, J. Chen, X. Fan, P. K. Yang, F. Yi and Z. L. Wang, Triboelectric-PyroelectricPiezoelectric Hybrid Cell for High-Efficiency Energy-
Harvesting and Self-Powered Sensing, Adv. Mater., 2015, 27(14), 2340-2347.

255 C. C. Li, Z. F. Du, L. M. Li, H. C. Yu, Q. Wan and T. H. Wang, Surface-depletion controlled gas sensing of $\mathrm{ZnO}$ nanorods grown at room temperature, Appl. Phys. Lett., 2007, 91(3), 032101.

256 A. Kolmakov, D. O. Klenov, Y. Lilach, S. Stemmer and M. Moskovits, Enhanced gas sensing by individual $\mathrm{SnO}_{2}$ nanowires and nanobelts functionalized with Pd catalyst particles, Nano Lett., 2005, 5(4), 667-673.

257 A. Gurlo, Nanosensors: towards morphological control of gas sensing activity. $\mathrm{SnO}_{2}, \mathrm{In}_{2} \mathrm{O}_{3}, \mathrm{ZnO}$ and $\mathrm{WO}_{3}$ case studies, Nanoscale, 2011, 3(1), 154-165.

258 D. Chen, J. Xu, Z. Xie and G. Shen, Nanowires assembled $\mathrm{SnO}_{2}$ nanopolyhedrons with enhanced gas sensing properties, ACS Appl. Mater. Interfaces, 2011, 3(6), 21122117.

259 Y. Zhao, P. Deng, Y. Nie, P. Wang, Y. Zhang, L. Xing and $\mathrm{X}$. Xue, Biomolecule-adsorption-dependent piezoelectric output of ZnO nanowire nanogenerator and its application as self-powered active biosensor, Biosens. Bioelectron., 2014, 57, 269-275.

260 Y. Nie, P. Deng, Y. Zhao, P. Wang, L. Xing, Y. Zhang and $\mathrm{X}$. Xue, The conversion of PN-junction influencing the piezoelectric output of a $\mathrm{CuO} / \mathrm{ZnO}$ nanoarray nanogenerator and its application as a room-temperature self-powered active $\mathrm{H}_{2} \mathrm{~S}$ sensor, Nanotechnology, 2014, 25(26), 265501.

261 X. Xue, Y. Nie, B. He, L. Xing, Y. Zhang and Z. L. Wang, Surface free-carrier screening effect on the output of a $\mathrm{ZnO}$ nanowire nanogenerator and its potential as a selfpowered active gas sensor, Nanotechnology, 2013, 24(22), 225501.

262 M. Law, L. E. Greene, J. C. Johnson, R. Saykally and P. Yang, Nanowire dye-sensitized solar cells, Nat. Mater., 2005, 4(6), 455-459.

263 Q. Wan, Q. H. Li, Y. J. Chen, T. H. Wang, X. L. He, J. P. Li and C. L. Lin, Fabrication and ethanol sensing characteristics of ZnO nanowire gas sensors, Appl. Phys. Lett., 2004, 84(18), 3654-3656.

264 S. Chu, G. Wang, W. Zhou, Y. Lin, L. Chernyak, J. Zhao, J. Kong, L. Li, J. Ren and J. Liu, Electrically pumped waveguide lasing from $\mathrm{ZnO}$ nanowires, Nat. Nanotechnol., 2011, 6(8), 506-510.

265 M. C. Jeong, B. Y. Oh, M. H. Ham and J. M. Myoung, Electroluminescence from $\mathrm{ZnO}$ nanowires in $\mathrm{n}-\mathrm{ZnO}$ film/ ZnO nanowire array/p-GaN film heterojunction lightemitting diodes, Appl. Phys. Lett., 2006, 88(20), 202105.

266 C. Y. Lu, S. J. Chang, S. P. Chang, C. T. Lee, C. F. Kuo, H. M. Chang, Y. Z. Chiou, C. L. Hsu and I. C. Chen, Ultraviolet photodetectors with $\mathrm{ZnO}$ nanowires prepared on ZnO: Ga/glass templates, Appl. Phys. Lett., 2006, 89(15), 153101.

267 W. Wu, S. Bai, N. Cui, F. Ma, Z. Wei, Y. Qin and E. Xie, Increasing UV photon response of $\mathrm{ZnO}$ sensor with nanowires array, Sci. Adv. Mater., 2010, 2(3), 402-406. 
268 W. Weng, Q. Sun, Y. Zhang, H. Lin, J. Ren, X. Lu, M. Wang and $\mathrm{H}$. Peng, Winding aligned carbon nanotube composite yarns into coaxial fiber full batteries with high performances, Nano Lett., 2014, 14(6), 3432-3438.

269 J. Ren, Y. Zhang, W. Bai, X. Chen, Z. Zhang, X. Fang, W. Weng, Y. Wang and H. Peng, Elastic and wearable wire-shaped lithium-ion battery with high electrochemical performance, Angew. Chem., 2014, 126(30), 7998-8003.

270 C. G. Granqvist, Transparent conductors as solar energy materials: a panoramic review, Sol. Energy Mater. Sol. Cells, 2007, 91(17), 1529-1598.

271 J. C. Scott, J. H. Kaufman, P. J. Brock, R. DiPietro, J. Salem and J. A. Goitia, Degradation and failure of MEH-PPV lightemitting diodes, J. Appl. Phys., 1996, 79(5), 2745-2751.

272 A. R. Schlatmann, D. W. Floet, A. Hilberer, F. Garten, P. J. Smulders, T. M. Klapwijk and G. Hadziioannou, Indium contamination from the indium-tin-oxide electrode in polymer light-emitting diodes, Appl. Phys. Lett., 1996, 69(12), 1764-1766.

273 M. Zhang, S. Fang, A. A. Zakhidov, S. B. Lee, A. E. Aliev, C. D. Williams, K. R. Atkinson and R. H. Baughman, Strong, transparent, multifunctional, carbon nanotube sheets, Science, 2005, 309(5738), 1215-1219.

274 D. Zhang, K. Ryu, X. Liu, E. Polikarpov, J. Ly, M. E. Tompson and C. Zhou, Transparent, conductive, and flexible carbon nanotube films and their application in organic lightemitting diodes, Nano Lett., 2006, 6(9), 1880-1886.

275 E. Artukovic, M. Kaempgen, D. S. Hecht, S. Roth and G. Grüner, Transparent and flexible carbon nanotube transistors, Nano Lett., 2005, 5(4), 757-760.

276 K. I. Park, J. H. Son, G. T. Hwang, C. K. Jeong, J. Ryu, M. Koo, I. Choi, S. H. Lee, M. Byun, Z. L. Wang and K. J. Lee, Highly-efficient, flexible piezoelectric PZT thin film nanogenerator on plastic substrates, Adv. Mater., 2014, 26(16), 2514-2520.

277 Y. Zhang, Y. W. Tan, H. L. Stormer and P. Kim, Experimental observation of the quantum Hall effect and Berry's phase in graphene, Nature, 2005, 438(7065), 201204.

278 A. K. Geim and K. S. Novoselov, The rise of graphene, Nat. Mater., 2007, 6(3), 183-191.

279 M. Orlita, C. Faugeras, P. Plochocka, P. Neugebauer, G. Martinez, D. K. Maude, A. L. Barra, M. Sprinkle, C. Berger, W. A. De Heer and M. Potemski, Approaching the Dirac point in high-mobility multilayer epitaxial graphene, Phys. Rev. Lett., 2008, 101(26), 267601.

280 C. Lee, X. Wei, J. W. Kysar and J. Hone, Measurement of the elastic properties and intrinsic strength of monolayer graphene, Science, 2008, 321(5887), 385-388.

281 E. W. Hill, A. K. Geim, K. Novoselov, F. Schedin and P. Blake, Graphene spin valve devices, IEEE Trans. Magn., 2006, 42(10), 2694-2696.

282 N. Tombros, C. Jozsa, M. Popinciuc, H. T. Jonkman and B. J. Van Wees, Electronic spin transport and spin precession in single graphene layers at room temperature, Nature, 2007, 448(7153), 571-574.
283 Z. Liu, Q. Liu, Y. Huang, Y. Ma, S. Yin, X. Zhang, W. Sun and Y. Chen, Organic photovoltaic devices based on a novel acceptor material: graphene, Adv. Mater., 2008, 20(20), 3924-3930.

284 X. Wang, L. Zhi and K. Müllen, Transparent, conductive graphene electrodes for dye-sensitized solar cells, Nano Lett., 2008, 8(1), 323-327.

285 L. B. Kong, T. Li, H. H. Hng, F. Boey, T. Zhang and S. Li, Waste mechanical energy harvesting (I): piezoelectric effect, in Waste Energy Harvesting, Springer, Berlin, Heidelberg, 2014, pp. 19-133.

286 Z. L. Wang, Towards self-powered nanosystems: from nanogenerators to nanopiezotronics, Adv. Funct. Mater., 2008, 18(22), 3553-3567.

287 Z. L. Wang, Piezoelectric nanostructures: From growth phenomena to electric nanogenerators, MRS Bull., 2007, 32(02), 109-116.

288 M. Bruchez, M. Moronne, P. Gin, S. Weiss and A. P. Alivisatos, Semiconductor nanocrystals as fluorescent biological labels, Science, 1998, 281(5385), 2013-2016.

289 W. C. Chan and S. Nie, Quantum dot bioconjugates for ultrasensitive nonisotopic detection, Science, 1998, 281(5385), 2016-2018.

290 S. Wang, N. Mamedova, N. A. Kotov, W. Chen and J. Studer, Antigen/antibody immunocomplex from CdTe nanoparticle bioconjugates, Nano Lett., 2002, 2(8), 817-822.

291 C. Mah, I. Zolotukhin, T. J. Fraites, J. Dobson, C. Batich and B. J. Byrne, Microsphere-mediated delivery of recombinant AAV vectors in vitro and in vivo, Mol. Ther., 2000, 1, S239.

292 D. Pantarotto, C. D. Partidos, J. Hoebeke, F. Brown, E. D. Kramer, J. P. Briand, S. Muller, M. Prato and A. Bianco, Immunization with peptide-functionalized carbon nanotubes enhances virus-specific neutralizing antibody responses, Chem. Biol., 2003, 10(10), 961-966.

293 R. L. Edelstein, C. R. Tamanaha, P. E. Sheehan, M. M. Miller, D. R. Baselt, L. Whitman and R. J. Colton, The BARC biosensor applied to the detection of biological warfare agents, Biosens. Bioelectron., 2000, 14(10), 805-813.

294 J. M. Nam, C. S. Thaxton and C. A. Mirkin, Nanoparticlebased bio-bar codes for the ultrasensitive detection of proteins, Science, 2003, 301(5641), 1884-1886.

295 J. Ma, H. Wong, L. B. Kong and K. W. Peng, Biomimetic processing of nanocrystallite bioactive apatite coating on titanium, Nanotechnology, 2003, 14(6), 619.

296 A. De La Isla, W. Brostow, B. Bujard, M. Estevez, J. R. Rodriguez, S. Vargas and V. M. Castano, Nanohybrid scratch resistant coatings for teeth and bone viscoelasticity manifested in tribology, Mater. Res. Innovations, 2003, 7(2), 110-114.

297 R. S. Molday and D. Mackenzie, Immunospecific ferromagnetic iron-dextran reagents for the labeling and magnetic separation of cells, J. Immunol. Methods, 1982, 52(3), 353-367.

298 R. Weissleder, G. Elizondo, J. Wittenberg, C. A. Rabito, H. H. Bengele and L. Josephson, Ultrasmall superparamagnetic iron oxide: characterization of a new 
class of contrast agents for MR imaging, Radiology, 1990, 175(2), 489-493.

299 J. Yoshida and T. Kobayashi, Intracellular hyperthermia for cancer using magnetite cationic liposomes, J. Magn. Magn. Mater., 1999, 194, 176-184.

300 W. J. Parak, D. Gerion, T. Pellegrino, D. Zanchet, C. Micheel, S. C. Williams, R. Boudreau, M. A. Le Gros, C. A. Larabell and A. P. Alivisatos, Biological applications of colloidal nanocrystals, Nanotechnology, 2003, 14(7), R15.

301 Z. Li, R. Yang, M. Yu, F. Bai, C. Li and Z. L. Wang, Cellular level biocompatibility and biosafety of $\mathrm{ZnO}$ nanowires, $J$. Phys. Chem. C, 2008, 112(51), 20114-20117.

302 G. T. Hwang, H. Park, J. H. Lee, S. Oh, K. I. Park, M. Byun, H. Park, G. Ahn, C. K. Jeong, K. No and H. Kwon, Selfpowered cardiac pacemaker enabled by flexible single crystalline PMN-PT piezoelectric energy harvester, $A d v$. Mater., 2014, 26(28), 4880-4887.
303 C. Dagdeviren, B. D. Yang, Y. Su, P. L. Tran, P. Joe, E. Anderson, J. Xia, V. Doraiswamy, B. Dehdashti, X. Feng and $\mathrm{B}$. Lu, Conformal piezoelectric energy harvesting and storage from motions of the heart, lung, and diaphragm, Proc. Natl. Acad. Sci. U. S. A., 2014, 111(5), 1927-1932.

304 L. Lin, Y. Hu, C. Xu, Y. Zhang, R. Zhang, X. Wen and Z. L. Wang, Transparent flexible nanogenerator as selfpowered sensor for transportation monitoring, Nano Energy, 2013, 2(1), 75-81.

305 G. T. Hwang, M. Byun, C. K. Jeong and K. J. Lee, Flexible Piezoelectric Thin-Film Energy Harvesters and Nanosensors for Biomedical Applications, Adv. Healthcare Mater., 2015, 4(5), 646-658.

306 Y. Yang, S. Wang, Y. Zhang and Z. L. Wang, Pyroelectric nanogenerators for driving wireless sensors, Nano Lett., 2012, 12(12), 6408-6413. 